\title{
Practical Vector Dirty Paper Coding for MIMO Gaussian Broadcast Channels
}

\author{
Shih-Chun Lin Student Member, IEEE and Hsuan-Jung Su Member, IEEE
}

\begin{abstract}
Recently, the vector dirty paper coding (DPC) achievable rate region has been shown to be the capacity region of a multiple-input multiple-output Gaussian broadcast channel (MIMO GBC). With DPC, the multiuser interference noncausally known at the transmitter can be completely removed. In this paper, we present a vector DPC structure for MIMO GBC. It is a generalization of the single antenna superposition dirty paper coding for the scalar Gaussian dirty paper problem proposed by Bennatan et al. In a theoretical random code setting, this construction is shown to be able to achieve the promised rate performance of the MIMO GBC. We also implement it with existing vector quantizer and capacity-achieving channel coding. Combined with iterative decoding, a design example validates the effectiveness of our methods.
\end{abstract}

Index Terms-Dirty paper coding, MIMO Gaussian broadcast channel, side-information

\section{INTRODUCTION}

$\mathbf{M}$ ULTIPLE-INPUT multiple-output (MIMO) antenna technology can highly increase the transmission data rate or the error performance when the bandwidth is constrained. In this system, both the transmitters and the receivers are equipped with multiple antennas. Recently, multiuser networks equipped with MIMO have gained lots of attention. In multiuser MIMO systems, the multiple transmitters and receivers share the same communication medium and cause mutual interference to each other. However, cooperation between multiple transmitters and/or receivers is possible to deal with the interference. When transmitters cooperate and receivers do not, the channel scenario is called the MIMO broadcast channel [1]-[5]. In this scenario, the joint transmitter sends independent information to multiple receivers at the same time, and each receiver (user) is interested in decoding its own message. The other channel scenario is called the MIMO multiple access channel (MAC) [2], [5], where transmitters do not cooperate and receivers do. In this scenario, each transmitter represents a different user sending independent information, and the joint receiver must decode information from all users.

Manuscript received June 2006; revised December, 2006 and February, 2007. This work was supported by the National Science Council, Taiwan, R.O.C., under grant NSC 95-2219-E-002-005. The material in this paper was presented in part at the Annual Conference on Information Sciences and Systems, Baltimore, USA, March 2005, and the International Symposium on Information Theory and Its Applications, Seoul, South Korea, OctoberNovember, 2006.

The authors are with the Department of Electrical Engineering and Graduate Institute of Communication Engineering, National Taiwan University, Taipei, Taiwan, 10617 (e-mail: f89921044@ntu.edu.tw, hjsu@cc.ee.ntu.edu.tw).

Digital Object Identifier 10.1109/JSAC.2007.070908.
We consider the optimal coding scheme for the MIMO Gaussian broadcast channel (MIMO GBC) [1], where the receiver noise is Gaussian. The channel gain matrices of all users are assumed to be known at the joint transmitter. For fixed wireless applications, accurate channel feedback from the receivers to the transmitter is possible. The capacity region of the MIMO GBC has recently been shown to coincide with the achievable rate region when vector dirty paper coding (DPC) is utilized [1]. With vector DPC [2] [3] [6], the multi-user interference, known at the transmitter, can be pre-subtracted for a desired receiver to achieve the interference-free rate performance. The receiver can then decode message as if the interference is known at receiver and cancelled. Some works have been proposed for the MIMO GBC, such as the practical precoding schemes in [7]-[9], and [10] which directly uses the conventional superposition codes [11] in non-degraded channels. However, none of these suboptimal coding schemes guarantee the theoretically promised rate performance. Most of these works are based on the Tomlinson-Harashima precoding [12] (THP). Some of them work only when each receiver has one antenna. As a simplified version of DPC with a scalar quantizer, THP suffers from shaping, modulo and power losses [13] as compared to the DPC.

The main contribution of this paper is presenting a practical vector DPC structure. In a theoretical random code setting, our construction is shown to be optimal and able to achieve the promised rate performance of the MIMO GBC. Our coding scheme is a generalization of the scheme proposed in [14] for the scalar DPC channel. And it applies to the environment when each user has more than one receiver antennas. The key is applying properly designed linear transformations in the spatial domain. We also design an end-to-end realization of this coding structure, which consists of a powerful vector quantizer and a capacity-approaching channel code. We provide a more systematic and intuitive way than [14] for choosing the practical code implementation parameters. With the aids of iterative decoding, simulation results confirm the effectiveness of our methods. As demonstrated by our design example, the proposed code implementation performs only 1.1 $\mathrm{dB}$ away from the capacity. This performance gap is similar to that of the best codes designed for the scalar DPC channel [14]-[16]. The benefits of using good vector quantizers have also been shown by comparing vector quantization with scalar quantization. In our example with a perfect channel codebook, at most about $3 \mathrm{~dB}$ gain can be obtained when optimal vector quantization is used.

The paper organization is as follows. In Section II we introduce the system model and problem formulation. The 
vector DPC structure is presented in Section III. Section IV provides the code parameters selection of this code by performing a random-coding analysis. Theorem 1 and its corollary guarantee the optimality of this coding structure. The practical code design using existing coding structures and some related design issues are given in Section V. Detailed code implementation parameters selection and a design example are shown in Section VI. Finally, Section VII concludes this paper.

\section{SYSTEM MODEL}

Consider a MIMO GBC system with $K$ users and $M$ transmitter antennas whose total transmission power is constrained. The channel gains from the transmitter antennas to the receiver antennas are assumed known perfectly at both the joint transmitter and the receivers. The optimal coding scheme for this channel [2] will first specify the vector DPC achievable rate for each user by determining the channel input covariance matrices and encoding order for all users, then apply the vector DPC on each user's message. The matrices maximizing the sum rate can be found using the algorithms in [17], which utilize the duality between the MIMO GBC and MAC [2]. The sum of the coded signals of all users will be sent to all receivers. At the receivers, whether or how a user's signal will be interfered by the other users' signals is governed by the vector DPC encoding order. In general, the signals encoded earlier will be invisible to the signals encoded later, while the former will be interfered by the latter. In this paper, we will focus on the coding scheme for a user who already knows the previously encoded users' signals and tries to achieve the specified interference-free rate.

For conciseness, the two-user scenario is first considered before the extension to the case with an arbitrary number of users. Here we focus on the coding scheme for user 1 who is assigned the interference-free rate (i.e. this user is the last encoded). The received signal at this user's receiver, which has $N$ receiver antennas, can be written as *

$$
\mathbf{y}_{t}^{c}=\mathbf{H}^{c}\left(\mathbf{x}_{1, t}^{c}+\mathbf{x}_{2, t}^{c}\right)+\mathbf{n}_{t}^{c}, \quad t=1 \ldots T,
$$

where $t$ is the time index, $T$ is the number of vector symbols in the code block; $\mathbf{y}_{t}^{c} \in \mathbb{C}^{N \times 1}$ is the $t$ th received symbol, $\mathbf{x}_{k, t}^{c} \in \mathbb{C}^{M \times 1}, k=1,2$, is the $t$ th vector symbol for the message of user $k, \mathbf{H}^{c} \in \mathbb{C}^{N \times M}$ is the MIMO channel gain matrix which is assumed to be constant over a code block, and $\mathbf{n}_{t}^{c} \in \mathbb{C}^{N \times 1}$ is the additive Gaussian noise at the receiver where $\mathbf{n}_{t}^{c} \sim N_{\mathbb{C}}\left(0, \mathbf{I}_{N}\right)$. In the sequel, we will first show that with the transmission power constraint $P_{1}$ for user 1 , when $T \rightarrow \infty$, there exists a coding structure achieving the interference-free rate [2]

$$
C_{I F}=\log \frac{\left|\mathbf{H}^{c} \Sigma_{1}\left(\mathbf{H}^{\mathbf{c}}\right)^{\dagger}+\mathbf{I}_{N}\right|}{\left|\mathbf{I}_{N}\right|},
$$

where $\Sigma_{1}$ is the specified positive-semidefinite channel input covariance matrix satisfying the power constraint, namely,

*In this paper, the matrices and vectors are denoted in boldface. For matrix $\mathbf{G}, \operatorname{Tr}(\mathbf{G})$ denotes the trace; $\operatorname{Rk}(\mathbf{G})$ denotes the rank; $\mathbf{G}^{\mathrm{T}}$ and $\mathbf{G}^{\dagger}$ denote the transpose and conjugate transpose, respectively. $\mathbf{G}_{s}^{-1}$ and $\left|\mathbf{G}_{s}\right|$ are the inverse and determinant of a square matrix $\mathbf{G}_{s}$. And $\mathbf{I}_{n}$ denotes the identity matrix of dimension $n$.
$\operatorname{Tr}\left(\Sigma_{1}\right)=P_{1}$. A practical implementation is then demonstrated for this coding structure.

To simplify the presentation, we rewrite (1) in the equivalent real channel representation by concatenating the real and imaginary parts of the complex vectors for all $T$ vector symbols

$$
\mathbf{y}=\mathbf{H x}+\mathbf{s}+\mathbf{n},
$$

where $\mathbf{x}=\left(\mathbf{x}_{1}^{\mathrm{T}}, \ldots, \mathbf{x}_{T}^{\mathrm{T}}\right)^{\mathrm{T}}$ with $\mathbf{x}_{t}^{\mathrm{T}}=\left(\operatorname{Re}\left\{\mathbf{x}_{1, t}^{c}\right\}^{\mathrm{T}}, \operatorname{Im}\left\{\mathbf{x}_{1, t}^{c}\right\}^{\mathrm{T}}\right)^{\mathrm{T}}$. The noncasually known side-information at the transmitter $\mathbf{s}$, and the additive noise $\mathbf{n} \sim N_{\mathbb{R}}\left(0, \frac{1}{2} \mathbf{I}_{2 N T}\right)$, are the real channel equivalents of the multi-user interference $\mathbf{H}^{c} \mathbf{x}_{2, t}^{c}$ and receiver noise $\mathbf{n}_{t}^{c}$, respectively, obtained similarly as $\mathbf{x}$ from $\mathbf{x}_{1, t}^{c}$. The $2 N T \times 2 M T$ block-diagonal real channel matrix $\mathbf{H}$ consists of the same $2 N \times 2 M$ diagonal-block repeated $T$ times

$$
\mathbf{H}=\mathbf{I}_{T} \otimes\left(\left[\begin{array}{cc}
\operatorname{Re}\left\{\mathbf{H}^{c}\right\} & -\operatorname{Im}\left\{\mathbf{H}^{c}\right\} \\
\operatorname{Im}\left\{\mathbf{H}^{c}\right\} & \operatorname{Re}\left\{\mathbf{H}^{c}\right\}
\end{array}\right]\right),
$$

where $\otimes$ denotes the Kronecker product. We also form the equivalent counterpart of $\Sigma_{1}$ as

$$
\Sigma_{G}=\frac{1}{2} \mathbf{I}_{T} \otimes\left(\left[\begin{array}{cc}
\operatorname{Re}\left\{\Sigma_{1}\right\} & -\operatorname{Im}\left\{\Sigma_{1}\right\} \\
\operatorname{Im}\left\{\Sigma_{1}\right\} & \operatorname{Re}\left\{\Sigma_{1}\right\}
\end{array}\right]\right) .
$$

\section{VECTOR DIRTY PAPER CODING}

Basically, the random "binning" technique which divides a set of randomly constructed codewords into subsets ("bins"), was used in the capacity-achievement proof in dirty paper channels [6], [13]. Motivated by this idea, several practical schemes have been proposed for the scalar dirty paper channel, such as the nested-lattice based approach [13] [15] and the superposition coding based approach [14]. The nested-lattice based approach uses a fine channel coding lattice and a coarse quantization lattice to form the bins, and the coarse lattice must be nested in the fine lattice. This "nested" property requires the fine and coarse lattices to be designed simultaneously, which complicates the design. To relax this requirement, Bennatan et.al. [14] proposed superposition DPC, and proved that independent selection of the quantization code and the channel code, which bears information, can still achieve the capacity.

The basic idea of the superposition DPC in [14] and its relation to the binning technique are briefly described here. For simplicity, consider an interference channel with identity channel matrix

$$
\mathbf{y}=\mathbf{x}+\mathbf{s}+\mathbf{n} .
$$

To transmit a message, the transmitter first selects a codeword $\mathbf{c}_{\mathbf{c}} \in \mathcal{C}_{C}$ representing it, where $\mathcal{C}_{C}$ is the channel code. It then finds a quantization codeword $\mathbf{c}_{\mathbf{q}} \in C_{q}$ nearest (in terms of joint-typicality) to $\mathbf{c}_{\mathbf{c}}-\mathbf{s}$, where $C_{q}$ is the quantization code. Finally, the quantization error

$$
\mathbf{x}=\left(\mathbf{c}_{\mathbf{c}}-\mathbf{s}\right)-\mathbf{c}_{\mathbf{q}}=\mathbf{c}-\mathbf{s},
$$

where $\mathbf{c}=\mathbf{c}_{\mathbf{c}}-\mathbf{c}_{\mathbf{q}} \in C \triangleq\left\{C_{C}+C_{q}\right\}$ (assuming $C_{q}$ is symmetric and $-\mathbf{c}_{\mathbf{q}} \in C_{q}$ ), is transmitted. Note that $C$ is the superposition of $\mathcal{C}_{C}$ and $\mathcal{C}_{q}$. In other words, the encoder operation can be seen as searching a codeword $\mathbf{c}$ nearest to the interference $\mathbf{s}$ over the coset code (bin) $\mathbf{c}_{\mathbf{c}}+C_{q}$. A good quantization code can be selected to make the quantization error meet the transmission 
power constraint. When the quantization code is a uniform scalar quantizer, the transmitter operation reduces to the THP [12]. From (5) and (6),

$$
\mathbf{y}=\mathbf{c}+\mathbf{n}
$$

and the interference is cancelled by pre-subtraction. The decoder searches in the superposition code $C$ to find the codeword $\hat{\mathbf{c}}=\hat{\mathbf{c}}_{\mathbf{c}}-\hat{\mathbf{c}}_{\mathbf{q}}$ that is nearest to $\mathbf{y}$. It then determines the unique coset (bin) $\hat{\mathbf{c}}_{\mathbf{c}}+\mathcal{C}_{q}$ to which $\hat{\mathbf{c}}$ belongs, and declares the decoded message to be the one represented by $\hat{\mathbf{c}}_{\mathbf{c}}$.

In the following, we introduce formally the vector superposition DPC. Unlike the channel in [14], the channel in (3) is similar to the Gaussian "colored-paper" channel [18] with the transmission power constraint $P_{1}$. Following the approach in [14], we consider a vector DPC for this channel consisting of a $\left(2^{T R_{q}}, m T\right)$ quantization code $C_{q}$ with rate $R_{q}$ and length $m T$, which makes the transmitted signal meet the total energy constraint $P_{1} T$; and a $\left(2^{T R_{c}}, n T\right)$ channel code $C_{c}$ with length $n T$, which boosts up the code rate $R_{c}$ to $C_{I F}$ in (2). Note that $T$ is the number of vector channel uses per code block, and $m, n$ are the numbers of the quantization and channel encoders output symbols per vector channel use, respectively. The codeword lengths of the channel code $n T$ and quantization code $m T$ will be determined in Section IV (equations (17) and (13)).

Before introducing the encoding/decoding procedures, we first define some useful operations. For a vector $\mathbf{g}$, the mod$A$ operation $(\operatorname{g} \bmod A)=\mathbf{g}^{\prime}$ is applied element-wise to each element $g_{i}$ of $\mathbf{g}$ such that $g_{i}{ }^{\prime}=g_{i}-Q_{A}\left(g_{i}\right) \forall i$, where $Q_{A}\left(g_{i}\right)$ is the nearest multiple of $A$ to $g_{i}$. The modulo operation associated with the quantization code $\mathcal{C}_{q}$ is defined as $\lfloor\mathbf{g}\rfloor C_{q} \triangleq$ $\left(\mathbf{g}-Q_{C_{q}}(\mathbf{g})\right) \bmod A$, where the $\bmod -A$ nearest neighbor quantizer $Q_{C_{q}}(\mathbf{g})$ associated with $C_{q}$ is defined by

$$
\begin{aligned}
& Q_{C_{q}}(\mathbf{g})=\mathbf{c} \in C_{q}, \quad \text { such that } \\
& \|(\mathbf{g}-\mathbf{c}) \bmod A\|^{2} \leq\left\|\left(\mathbf{g}-\mathbf{c}^{\prime}\right) \bmod A\right\|^{2}, \quad \forall \mathbf{c}^{\prime} \in \mathcal{C}_{q}
\end{aligned}
$$

where $\|\cdot\|$ denotes the Euclidean norm. As in [14], the mod$A$ operation is borrowed from the construction- $A$ approach which generates lattices from linear codes. Its effect can be equivalently modeled as the tessellation of the entire real number space $\mathbb{R}^{m T}$ with replicas of the cube $[-A / 2, A / 2]^{m T}$. The mod- $A$ operation in the superposition DPC serves a different purpose compared to the modulo-lattice operation of the nested-lattice DPC. The reader is referred to [14, Sec. III.C] for more discussions on the connection between these two coding schemes and the mod- $A$ operation.

The encoder and decoder structures of the vector DPC for MIMO GBC are summarized as follows.

Encoder: The encoder selects a codeword $\mathbf{c}_{\mathbf{c}} \in C_{c}$ according to the message index and transmits the vector

$$
\mathbf{x}=\mathbf{F}_{\mathbf{q}} \cdot\left\lfloor\left(\mathbf{F}_{\mathbf{c}} \mathbf{c}_{\mathbf{c}}-\mathbf{W s}-\mathbf{u}\right) \bmod A\right\rfloor_{C_{q}},
$$

where $\mathbf{u}$, uniformly distributed in the $m T$-dimensional cube $[-A / 2, A / 2]^{m T}$ and independent of the channel, is a dither signal known to both the transmitter and the receiver. The dimensions of the linear transformations $\mathbf{F}_{\mathbf{q}}, \mathbf{F}_{\mathbf{c}}$ and $\mathbf{W}$ are $2 M T \times m T, m T \times n T$ and $m T \times r T$, respectively, where we

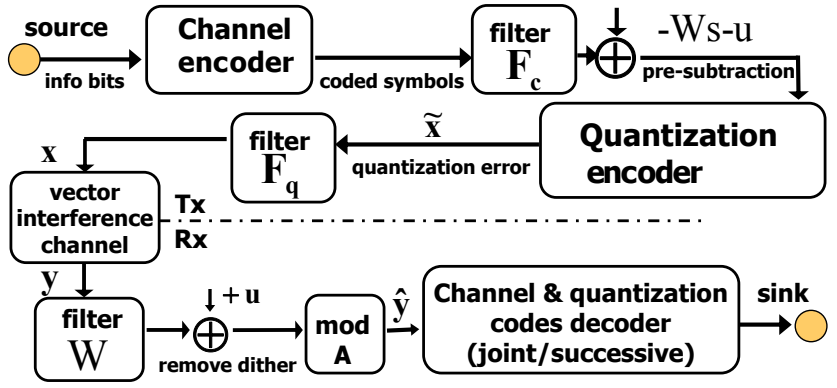

Fig. 1. Schematic description of the proposed vector dirty paper coding structure

define $r \triangleq 2 N$ to simplify notations. The roles and selections of these transformations will be clarified in Section IV. And the transmitted signal $\mathbf{x}$ must meet the power constraint $\|\mathbf{x}\|^{2} / T \leq$ $P_{1}$.

Before describing the decoder, we first provide some helpful formulae. Similar to [14], the transmitted signal $\mathbf{x}$ can also be expressed as

$$
\begin{aligned}
\mathbf{x} & =\mathbf{F}_{\mathbf{q}}\left(\left(\mathbf{c}_{\mathbf{q}}+\mathbf{F}_{\mathbf{c}} \mathbf{c}_{\mathbf{c}}-\mathbf{W s}-\mathbf{u}\right) \bmod A\right) \\
& =\mathbf{F}_{\mathbf{q}} \tilde{\mathbf{x}},
\end{aligned}
$$

where $\mathbf{c}_{\mathbf{q}}=Q_{C_{q}}\left(\left(-\mathbf{F}_{\mathbf{c}} \mathbf{c}_{\mathbf{c}}+\mathbf{W s}+\mathbf{u}\right) \bmod A\right)$ is the quantizer output and $\tilde{\mathbf{x}}$ is the quantization error with a mod- $A$ distortion measure. The quantization code to be specified in Section $\mathrm{V}$ will make both $\tilde{\mathbf{x}}$ and $\mathbf{x}$ zero-mean. By defining an equivalent channel $\tilde{\mathbf{H}} \triangleq \mathbf{H F}_{\mathbf{q}}$ for $\tilde{\mathbf{x}}$, the channel (3) can be recast as

$$
\mathbf{y}=\tilde{\mathbf{H}} \tilde{\mathbf{x}}+\mathbf{s}+\mathbf{n} .
$$

In this encoder structure, the transmitted signal is the superposition of the linearly transformed codewords from the two codebooks $C_{q}$ and $\mathcal{C}_{c}$. As will be shown in Section IV ( equations (12) and (16) ), the linear transformations $\mathbf{F}_{\mathbf{q}}$ and $\mathbf{F}_{\mathbf{c}}$ are full column-rank and one-to-one. When we transform the codebook $C_{q}$ with $\mathbf{F}_{\mathbf{q}}$ to form a new codebook $C_{q}^{\prime}$, the superposition codeword $\mathbf{F}_{\mathbf{q}} \mathbf{c}_{\mathbf{q}}+\mathbf{F}_{\mathbf{q}} \mathbf{F}_{\mathbf{c}} \mathbf{c}_{\mathbf{c}}$ belongs to the coset of $C_{q}^{\prime}$. This is consistent with the design guidelines in [6].

Decoder: After receiving $\mathbf{y}$, the decoder first performs signal processing on $\mathbf{y}$ to get

$$
\begin{aligned}
\hat{\mathbf{y}} & =(\mathbf{W y}+\mathbf{u}) \bmod A \\
& =((\tilde{\mathbf{x}}+\mathbf{W} \mathbf{s}+\mathbf{u})+\mathbf{e}) \bmod A \\
& =\left(\mathbf{F}_{\mathbf{c}} \mathbf{c}_{\mathbf{c}}+\mathbf{c}_{\mathbf{q}}+\mathbf{e}\right) \bmod A,
\end{aligned}
$$

where $\mathbf{e}=-\left(\mathbf{I}_{m T}-\mathbf{W} \tilde{\mathbf{H}}\right) \tilde{\mathbf{x}}+\mathbf{W n}$ and the second equality comes from (9) and the distributive property of the mod- $A$ operation, and $\mathbf{W}$ acts as an equalizer. We can jointly decode $\left(\mathbf{c}_{\mathbf{c}}, \mathbf{c}_{\mathbf{q}}\right)$, or perform successive decoding on $\hat{\mathbf{y}}$. The latter means that we first decode $\hat{\mathbf{c}_{\mathbf{q}}}$ from $\hat{\mathbf{y}}$, then subtract it from $\hat{\mathbf{y}}$ and decode the desired codeword $\hat{\mathbf{c}_{\mathbf{c}}}$ from $\left(\hat{\mathbf{y}}-\hat{\mathbf{c}_{\mathbf{q}}}\right) \bmod A$.

The proposed encoder/decoder structure is shown in Fig. 1. The messages are encoded by the channel encoder. After filtering the channel-coded symbols with $\mathbf{F}_{\mathbf{c}}$, the encoder pre-subtracts the filtered interference Ws and the dither $\mathbf{u}$. The resulted signal is fed to the quantization encoder. The quantization encoder outputs its quantization error $\tilde{\mathbf{x}}$. Finally 
the transmitter filters this quantization error with $\mathbf{F}_{\mathbf{q}}$ and sends it to the channel. At the receiver, after forming $\hat{\mathbf{y}}$ according to (11), the decoder decodes the messages in $\hat{\mathbf{y}}$. Since $\hat{\mathbf{y}}$ consists of two codewords, the message-bearing channel codeword $\mathbf{c}_{\mathbf{c}}$ and the quantization codeword $\mathbf{c}_{\mathbf{q}}$ which is independent of the messages, we can recover the messages by either jointly decoding them or decoding $\mathbf{c}_{\mathbf{q}}$ first then decoding $\mathbf{c}_{\mathbf{c}}$.

\section{Code Parameters Selection and the INTERFERENCE-FREE RATE ACHIEVEMENT}

We now describe how to choose the filters $\mathbf{F}_{\mathbf{q}}, \mathbf{F}_{\mathbf{c}}, \mathbf{W}$, the quantization code rate $R_{q}$, and the modulo-size $A$ that can make $R_{c}$ achieve $C_{I F}$ while satisfying the energy constraint $P_{1} T$. The transmitter matrix filter $\mathbf{F}_{\mathbf{q}}$ must satisfy

$$
\mathbf{F}_{\mathbf{q}} \mathbf{F}_{\mathbf{q}}^{\mathrm{T}}=\Sigma_{G}
$$

where $\Sigma_{G}$ is defined in (4). The purpose of this filter is to shape the quantization error $\tilde{\mathbf{x}}$ to fit the given channel input covariance matrix. Since $\Sigma_{1}$ is positive semidefinite, $\Sigma_{G}$ will also be positive semidefinite. We can always find a $2 M T \times$ $\operatorname{Rk}\left(\Sigma_{G}\right)$ full column rank matrix $\mathbf{F}_{\mathbf{q}}$ that meets (12) [19]. The quantization codeword length is chosen as

$$
m T=\operatorname{Rk}\left(\Sigma_{G}\right) .
$$

Note that $\operatorname{Rk}\left(\Sigma_{G}\right)$ is a multiple of $T$. When $\Sigma_{1}$ is not full rank, $m / 2<M$, the required dimension of the quantization codeword per complex channel use is smaller than the number of transmit antennas. When $\Sigma_{1}$ is positive definite, $\mathbf{F}_{\mathbf{q}}$ can be found by performing Choletsky factorization on $\Sigma_{G}$, and it is nonsingular and lower triangular. Otherwise, $\Sigma_{1}$ is singular and $\mathbf{F}_{\mathbf{q}}$ can be found by performing eigenvalue decomposition on $\Sigma_{G}$. It is only full column rank and singular.

For the equivalent channel (10), we select the equalizer $\mathbf{W}$ to be the linear minimum mean square error (LMMSE) equalizer to estimate $\tilde{X}_{G}^{m}$ given $Y_{f}^{r}$ in the following channel

$$
Y_{f}^{r}=\tilde{\mathbf{H}} \tilde{X}_{G}^{m}+N^{r},
$$

where $\tilde{X}_{G}^{m} \sim N_{\mathbb{R}}\left(0, \mathbf{I}_{m T}\right)$, and $N^{r} \sim N_{\mathbb{R}}\left(0, \frac{1}{2} \mathbf{I}_{r T}\right)$ has the same distribution as $\mathbf{n}$. Using a similar approach as in [3, equations (22)-(24)], it can be shown that

$$
\mathbf{W}=\left(\tilde{\mathbf{H}}^{\mathrm{T}} \tilde{\mathbf{H}}+\frac{1}{2} \mathbf{I}_{m T}\right)^{-1} \tilde{\mathbf{H}}^{\mathrm{T}} .
$$

The matrix filter $\mathbf{F}_{\mathbf{c}}$ must satisfy

$$
\mathbf{F}_{\mathbf{c}} \mathbf{F}_{\mathbf{c}}^{\mathrm{T}}=\mathbf{W} \tilde{\mathbf{H}} \text {. }
$$

This filter plays an important role to adjust the equivalent noise in the successive decoding (detailed in Section IV-A) such that reliable decoding is possible. Substituting (15) in $\mathbf{W} \tilde{\mathbf{H}}$, it is easy to see that $\mathbf{W} \tilde{\mathbf{H}}$ is always positive semidefinte so a full column rank matrix $\mathbf{F}_{\mathbf{c}}$ can be found with the same procedure used to find $\mathbf{F}_{\mathbf{q}}$. The number of columns of $\mathbf{F}_{\mathbf{c}}$, which equals to the rank of $\mathbf{W} \tilde{\mathbf{H}}$, can be used to determine the channel codeword length $n T$. According to (15), $\operatorname{Rk}(\mathbf{W} \tilde{\mathbf{H}})$ equals to $\operatorname{Rk}\left(\tilde{\mathbf{H}}=\mathbf{H F}_{\mathbf{q}}\right)$. We then choose $n T$ as

$$
n T=\operatorname{Rk}(\tilde{\mathbf{H}}) \leq \min \left(\operatorname{Rk}(\mathbf{H}), \operatorname{Rk}\left(\mathbf{F}_{\mathbf{q}}\right)=m T\right) .
$$

With these filters, we use randomly generated codebooks to find the remaining code parameters, and summarize the result in the following theorem.

Theorem 1: With filters $\mathbf{F}_{\mathbf{q}}, \mathbf{W}$ and $\mathbf{F}_{\mathbf{c}}$ chosen as in (12), (15) and (16), respectively, and $T \rightarrow \infty$, there exists codebooks $C_{q}$ with codeword length $m T$ given in (13) and $C_{c}$ with codeword length $n T$ given in (17) such that

- The encoder error probability (when the power constraint is violated) approaches zero, if the quantization code rate satisfies

$$
R_{q}>(2 \tilde{M} \log A-\tilde{M} \log (2 \pi e))+\delta_{1}=R_{q}^{o}+\delta_{1},
$$

where $\tilde{M} \triangleq m / 2$,

- The decoder error probability (when jointly decoding $\mathbf{c}_{\mathbf{q}}$ and $\mathbf{c}_{\mathbf{c}}$ ) approaches zero, if

$$
\begin{gathered}
R_{c}+R_{q} \leq C_{I F}+R_{q}^{o}+\delta_{3}, \\
R_{c} \leq C_{I F}+\delta_{2}
\end{gathered}
$$

where $\delta_{1}, \delta_{2}, \delta_{3} \rightarrow 0$ as $A \rightarrow \infty$, and $C_{I F}$ is defined in (2). Thus, as $A \rightarrow \infty$ the interference-free channel coding rate is achievable at $\left(R_{q}, R_{c}\right)=\left(R_{q}^{o}, C_{I F}\right)$ with joint decoding. Moreover, it is also achievable with successive decoding.

Proof: In Appendix I.

In this theorem, $A$ must be very large compared to the variance of the quantization error $\tilde{\mathbf{x}}$ (per codeword element). This does not mean that the mod- $A$ operation is not working in practice. Even if we choose a large $A$, the dynamic range of the interference $\mathbf{s}$ may exceeds it and the $\bmod -A$ operation will limit the quantizer input range to make the encoder work. In Section V, we will discuss how large $A$ has to be when the existing structured codebooks are used.

As in [14], in the random-coding based proof of Theorem 1 , the encoder-decoder pair uses the joint-typicality instead of the minimum-distance metrics to find the codewords. This is due to the "self-noise" term $\tilde{\mathbf{x}}$ in e of (11), which might be correlated with the quantizer output $\mathbf{c}_{\mathbf{q}}$. The readers are referred to [14] for detailed discussion.

Intuitively, the power constraint $P_{1}$ is met due to the following reasons. Observing the encoder, we in fact transmit the linearly-transformed quantization error $\mathbf{F}_{\mathbf{q}} \tilde{\mathbf{x}}$. According to [20], a good vector quantizer can make its quantization error distribution similar to the one specified in the rate-distortion test channel [11]. Therefore, if we choose the covariance matrix of the quantization error in the test channel as an identity matrix, and $R_{q}$ close to the rate-distortion bound, the selection in (12) will make the covariance matrix of $\mathbf{x}=\mathbf{F}_{\mathbf{q}} \tilde{\mathbf{x}}$ equal to $\Sigma_{G}$. Then the transmitter will meet the constraint since $\operatorname{Tr}\left(\Sigma_{G}\right) / T=P_{1}$. Note that the distortion measure in (7) belongs to the mod- $\Lambda$ difference-distortion measure defined in [21] if $\Lambda=A \mathbf{Z}^{m T}$. The rate-distortion bound is achieved if the quantization error distribution is chosen as the truncated Gaussian distribution [21]. Although we let the quantization error be Gaussian in our proof, Gaussian distribution approaches truncated Gaussian distribution as $A \rightarrow \infty$, and $R_{q}^{o}$ is the ratedistortion bound. 


\section{A. Feasibility of successive decoding}

The decoder can perform successive decoding to reduce the complexity. We now verify the capacity achievability of successive decoding. Since the concept is easier to explain with the random code setting used in Appendix I, the following MAC channel corresponding to (11) is considered

$$
\hat{Y}^{m}=\left(U_{q}^{m}+\mathbf{F}_{\mathbf{c}} U_{c}^{n}+E^{m}\right) \bmod A,
$$

where $U_{q}^{m}$ and $U_{c}^{n}$ are random vectors with dimensions $m T$ and $n T$ used to generate the random counterparts of codebooks $C_{q}$ and $\mathcal{C}_{c}$, respectively. According to the proof in Appendix I-B, W will make $-E^{m}$ equal to the MMSE estimation error of $\tilde{X}_{G}^{m}$ in the channel (14). For easier explanation, in the following we assume $A \rightarrow \infty$ so the module operations can be omitted at the decoder. A rigorous proof of the feasibility of successive decoding can be found in Appendix I-B.

Since the quantization code is transmitted with rate $R_{q}^{o}$, it must be no larger than the maximum reliable rate $I\left(U_{q}^{m} ; \hat{Y}^{m}\right)$ when decoding the quantization codeword from $\hat{Y}^{m}$ with noise $\mathbf{F}_{\mathbf{c}} U_{c}^{n}+E^{m}$, where $I(;)$ denotes the mutual information between random vectors. The rate-distortion lower bound $R_{q}^{o}$ is the mutual information between the quantizer output $U_{q}^{m}$ and input ( $U_{q}^{m}$ plus a white Gaussian quantization error). As the filters $\mathbf{F}_{\mathbf{c}}$ and $\mathbf{W}$ selected previously can make $\mathbf{F}_{\mathbf{c}} U_{c}^{n}+$ $E^{m}$ a white Gaussian random vector, which equals to the quantization error in distribution, $R_{q}^{o}=I\left(U_{q}^{m} ; \hat{Y}^{m}\right)$ and errorfree decoding of the quantization code is possible. Indeed, the duality between source and channel coding [11] has been invoked between the quantization test channel and the channel between $U_{q}^{m}$ and $\hat{Y}^{m}$. The filters selected establish the equivalence between these two channels and make reliable decoding possible.

After subtracting the correctly decoded quantization codeword from (20), the decoder decodes the channel code in

$$
\left(\mathbf{F}_{\mathbf{c}} U_{c}^{n}+E^{m}\right) \bmod A,
$$

The selected filters will make this channel similar to a modulo backward-channel of the MMSE estimation [22] of (14). The achievable rate $C_{I F}$ of the corresponding interferencefree forward channel (14) is thus achievable by this channel (21) using the information-lossless property of the MMSE estimation. Indeed, the filter $\mathbf{W}$ corresponds to the MMSEfactor $\alpha$ in the "inflated lattice" proposed in [23]. Similar principle has also been used in other MIMO channels [24], except that in [24] a whitening filter has been applied to the signal $\mathbf{W y}+\mathbf{u}$ to whiten the MMSE estimation error for lattice decoding. Furthermore, our filter selection criteria can also be combined with nested-lattice encoding/decoding to achieve the interference-free rate [25], which can be seen as a vector generalization of the scalar DPC in [13] [23]. And the encoding structure in this paper can be treated as a variation of the lattice-based vector DPC in [25], when the quantization and channel coding lattices are both construction$A$. This is inherited from the relationship between the scalar nested-lattice DPC [13] and the superposition DPC [14].

\section{B. Extension to more than two users}

Finally, we extend the results we obtained to the situation with more than two users. Let user $k$ in a $K$-user system be assigned a channel input covariance matrix $\Sigma_{k}$ and power constraint $P_{k}=\operatorname{Tr}\left(\Sigma_{k}\right)$. The total transmitter power constraint is $\sum_{k=1}^{K} P_{k}$. Without loss of generality, we focus on user $j$ and assume it is encoded after all the users with indexes larger than $j$, and is going to cancel the interference from these users. In the following, all parameters with subscript $j$ indicate the corresponding parameters defined previously. The $t$ th received complex symbol for this user is

$$
\mathbf{y}_{j, t}^{c}=\mathbf{H}_{j}^{c} \mathbf{x}_{j, t}^{c}+\mathbf{H}_{j}^{c}\left(\sum_{k=j+1}^{K} \mathbf{x}_{k, t}^{c}\right)+\left(\mathbf{n}_{t}^{c}+\mathbf{H}_{j}^{c}\left(\sum_{k=1}^{j-1} \mathbf{x}_{k, t}^{c}\right)\right) .
$$

By performing the same procedure as in Section II, we rewrite the above channel as the equivalent real interference channel

$$
\mathbf{y}_{j}=\mathbf{H}_{j} \mathbf{x}_{j}+\mathbf{s}_{j}+\mathbf{n}_{j},
$$

where $\mathbf{s}_{j}$ is the interference to be cancelled and the noise term $\mathbf{n}_{j}$ is the sum of the additive noise and the residual multi-user interference from signals with user indexes smaller than $j$. The covariance matrix of $\mathbf{n}_{j}$ is denoted by $\Sigma_{n_{j}}$ and it is positive definite. The vector DPC described in Section III can be easily adopted for this case by changing the noise covariance matrix from $\frac{1}{2} \mathbf{I}_{2 N_{j} T}$ to $\Sigma_{n_{j}}$. The corresponding LMMSE filter $\mathbf{W}_{j}$ is chosen as

$$
\mathbf{W}_{j}=\left(\mathbf{I}_{m_{j} T}+\tilde{\mathbf{H}}_{j}^{\mathrm{T}} \Sigma_{n_{j}}^{-1} \tilde{\mathbf{H}}_{j}\right)^{-1} \tilde{\mathbf{H}}_{j}^{\mathrm{T}} \Sigma_{n_{j}}^{-1} .
$$

The channel code length of this user is $\operatorname{Rk}\left(\tilde{\mathbf{H}}_{j}\right)$. We then have the following corollary, whose proof is given in Appendix I-C.

Corollary 1: If the modulo-size $A_{k}$ for user $k$ 's quantizer approaches infinity for all the $K$ users, with $\mathbf{W}_{j}$ specified in (22) and all other code parameters selected as in Theorem 1 , for user $j$ under power constraint $P_{j}$, the following rate is achievable with the proposed vector dirty paper coding

$$
R_{c, j}=\log \frac{\left|\mathbf{I}_{N_{j}}+\sum_{k \leq j} \mathbf{H}_{j}^{c} \Sigma_{k}\left(\mathbf{H}_{j}^{c}\right)^{\dagger}\right|}{\left|\mathbf{I}_{N_{j}}+\sum_{k<j} \mathbf{H}_{j}^{c} \Sigma_{k}\left(\mathbf{H}_{j}^{c}\right)^{\dagger}\right|} .
$$

If we form a vector DPC rate vector $\left(R_{c, 1}, \ldots R_{c, K}\right)^{\mathrm{T}}$, where $R_{c, j}$ is as specified in (23), the capacity region of the MIMO GBC [1], [2] is the convex hull of the union of this vector over all user orderings and all positive semidefinite channel input covariance matrices obeying the total power constraint.

\section{Practical Design Issues and Coding Structures}

In this section, we use some existing coding structures to design the quantization and channel codes. Some design issues related to the practical code selection are presented in the first two subsections, and the final overall coding block diagram is given in the last subsection. The vector quantizer is selected first. According to the proof of Theorem 1, the random vector $U_{q}^{m}$ used to generate the random quantization codebook is uniformly distributed in the hypercube $[-A / 2, A / 2]^{m T}$. The trellis-coded quantization (TCQ) [26] is a good candidate to implement this random codebook. Its support region is a hypercube and most trellis codes are geometrically uniform [27]. As for the modulo-size $A$, first note that due to the dither $\mathbf{u}$, the quantizer input in (8) is also uniformly distributed 
in $[-A / 2, A / 2]^{m T}$ and has variance $A^{2} / 12$ (per codeword element) according to [28, Lemma 1]. Since the variance of the quantization error $\tilde{\mathbf{x}}$ is unity per codeword element from the proof of Theorem I, we select the modulo size $A$ satisfying

$$
D_{q} \cdot\left(A^{2} / 12\right)=1,
$$

where $D_{q}$ is the inverse of the quantizer-input to quantizationerror ratio [26] of the TCQ. Based on this selected A, we will discuss the performance loss due to the difference between the TCQ code rate and the optimal code rate $R_{q}^{o}$ in Theorem 1. Comparison with the scheme using a scalar quantizer will be presented. In the following derivations, we assume that the number of trellis states of the TCQ is large enough to make the TCQ output a good approximation of $U_{q}^{m}$, and the quantization error distribution approximately Gaussian.

As for the channel code selection, also from the proof of Theorem 1, the random vector $U_{c}^{n}$ used to generate the random channel codebook has a white Gaussian distribution. This is the same as the capacity-achieving random codes for the AWGN channel and the single user ergodic MIMO channel without channel feedback. The nonsystematic irregular repeat-accumulate (IRA) code [29] with proper finitealphabet constellation is adopted. This code has been shown to approach the capacity in the above channels [30] and the scalar dirty-paper channel [15]. The low-density party check (LDPC) code adopted in [14] is another choice. However, the IRA encoder has lower complexity compared with the LDPC encoder. We will show the impact of finite constellation size with perfect channel coding in Section V-B. The IRA code will introduce an additional loss, which can be reduced by properly designing its edge distribution, as will be shown in Section VI-B. According to the selected TCQ and IRA codes, the proposed end-to-end vector dirty paper coding structure is shown in Section V-C.

\section{A. Performance loss due to the practical quantization code}

Compared with the rate-distortion bound achieving codebook required in Theorem 1, using practical TCQ introduces a performance loss. To estimate this loss, we consider $C_{I F}+R_{q}^{o}=I\left(U_{q}^{m}, \mathbf{F}_{\mathbf{c}} U_{c}^{n} ; \hat{Y}^{m}\right) / T$, the maximum sum rate $\hat{Y}^{m}$ in (20) can support. First rewrite $R_{q}^{o}$ in (18) with $D_{q}$ described previously,

$$
R_{q}^{o}=\tilde{M} \log \left(\frac{12}{2 \pi e D_{q}}\right) .
$$

From discussions of Theorem 1, $A$ must be large enough to make $R_{q}^{o}$ equal to the rate-distortion bound. Typically, the $D_{q}$ of conventional TCQ makes $A$ several times the unit variance (per element) of the quantization error, as in [31, P.826]. The assumption is thus valid. Otherwise, we can increase the TCQ code rate or number of trellis states to lower $D_{q}$, and make $A$ larger according to our selection method (24). In practice, to achieve a particular $D_{q}$, the code rate $R_{q}^{T}$ of the TCQ should always be larger than the optimal quantization code rate $R_{q}^{o}$. If the TCQ is good enough to meet our assumptions, (19) can be used as an indicator of its impact on the receiver performance. From (19), we know that using a TCQ with code rate $R_{q}^{T}$, there will be an achievable channel code rate loss $R_{q}^{T}-R_{q}^{o}$.
Interestingly, a similar performance loss was reported for the vector DPC based on lattice codes proposed in [25]. To see this, for lattice-based vector DPC, non-ideal white quantization lattice code will produce a rate loss of

$$
\tilde{M} \log \left(2 \pi e G\left(\Lambda_{q}\right)\right)
$$

where $G\left(\Lambda_{q}\right)$ is the normalized second-order moment of this quantization lattice [25]. Similar normalized second-order moment $G\left(C_{q}\right)$ of the TCQ [27] can be approximated as

$$
G\left(C_{q}\right) \simeq 1 /\left(\frac{A^{m T}}{2^{R_{q}^{T} T}}\right)^{\frac{2}{m T}}=\frac{D_{q}}{12} 2^{R_{q}^{T} / \tilde{M}},
$$

due to the unit variance of the quantization error and the fact that TCQ is geometrically uniform in the hypercube. The rate loss $R_{q}^{T}-R_{q}^{o}$ is then

$$
R_{q}^{T}-\tilde{M} \log \left(\frac{12}{2 \pi e D_{q}}\right) \simeq \tilde{M} \log \left(2 \pi e G\left(C_{q}\right)\right),
$$

which coincides with (26).

For comparison, we also investigate the performance when a scalar quantizer is used. The transmitter is

$$
\mathbf{x}=\mathbf{F}_{\mathbf{q}} \cdot\left(\left(\mathbf{c}_{\mathbf{c}}-\mathbf{W} \mathbf{s}-\mathbf{u}\right) \bmod A\right) .
$$

With $\mathbf{F}_{\mathbf{q}}$ meeting (12), $A$ must satisfy $A^{2} / 12=1$. The received signal after signal processing (11) becomes

$$
\hat{\mathbf{y}}=\left(\mathbf{c}_{\mathbf{c}}+\mathbf{e}\right) \bmod A .
$$

We again consider the random coding setting to find the maximum supportable rate $I\left(U_{c}^{m} ; \hat{Y}^{m}\right) / T$. To do this, we let the random vector $U_{c}^{m}$ in Section IV be uniformly distributed in $[-A / 2, A / 2]^{m T}$ and independent of the dither and interference according to [31]. Note that in (29) $\mathbf{F}_{\mathbf{c}}$ is set as an identity matrix $(m=n)$. If it is not, $\hat{\mathbf{y}}$ will become $\left(\mathbf{F}_{\mathbf{c}} \mathbf{c}_{\mathbf{c}}+\mathbf{e}\right) \bmod A$ and the optimal $U_{c}^{m}$ must make $\mathbf{F}_{\mathbf{c}} U_{c}^{m}$ uniformly distributed in $[-A / 2, A / 2]^{m T}$. In this case, the maximum $I\left(U_{c}^{m} ; \hat{Y}^{m}\right) / T$ remains the same. The equivalent noise $E^{m}$ consists of a random vector formed by linearly-transforming a vector uniformly distributed in $[-A / 2, A / 2]^{m T}$ with $-\left(\mathbf{I}_{m T}-\mathbf{W} \tilde{\mathbf{H}}\right)$ and a Gaussian vector $\mathbf{W n}$. In addition, $E^{m}$ is independent of the transmitted signal and $U_{c}^{m}$. The mutual information $I\left(U_{c}^{m} ; \hat{Y}^{m}\right)$ then becomes $m T \log A-h\left(E^{m} \bmod A\right)$. Though $E^{m}$ is far from Gaussian, its probability density function (PDF) can be calculated analytically through a convolution integral as in [15]. The maximum achievable rate $I\left(U_{c}^{m} ; \hat{Y}^{m}\right) / T$ can be obtained with numerical integrals. We will use this formula to calculate the performance loss and compare it with the proposed scheme utilizing TCQ.

\section{B. Maximum achievable channel code rate using finite alpha- bet constellation}

According to the proof of Theorem 1, the channel code should have a Gaussian constellation. However, finite-alphabet constellations such as pulse amplitude modulation (PAM) are often used in practice. The impacts of a finite $A$ also need to be investigated. To this end, we focus on the highest possible channel code rate of the channel (21). Some useful definitions are first defined. We first define a $n \times 1$ signal vector $\tilde{U}_{c}$ whose elements are independently and equally likely chosen from a 
finite constellation with the average power normalized to unity. Also, let $\Sigma_{E}$ denote the covariance matrix of $E^{m}$ in (21), which can be easily computed as in Appendix I-B. Since $\mathbf{F}_{\mathbf{c}}$ and $\Sigma_{E}$ in (21) are block-diagonal, we define a $m \times n$ matrix $\tilde{\mathbf{F}}_{\mathbf{c}}$ and a $m \times m$ matrix $\Sigma_{\tilde{E}}$, where $\mathbf{I}_{T} \otimes \tilde{\mathbf{F}}_{\mathbf{c}}=\mathbf{F}_{\mathbf{c}}$ and $\mathbf{I}_{T} \otimes \Sigma_{\tilde{E}}=\Sigma_{E}$, respectively. We also define $\tilde{E}$ as a $m \times 1$ zero-mean Gaussian random vector with covariance matrix $\Sigma_{\tilde{E}}$.

The maximum achievable rate when using a finite-alphabet constellation in channel (21) is

$$
h\left(\left(\tilde{\mathbf{F}}_{\mathbf{c}} \tilde{U}_{c}+\tilde{E}\right) \bmod A\right)-h(\tilde{E} \bmod A) .
$$

This is the best system performance with the optimal channel coding. Note that changing the distribution from Gaussian to $\tilde{U}_{c}$ does not change the sum rate (19), if the TCQ meets the assumptions described previously. Thus the results in the previous subsection are still valid. However, the differential entropy $h\left(\left(\tilde{\mathbf{F}}_{\mathbf{c}} \tilde{U}_{c}+\tilde{E}\right) \bmod A\right)$ in (30) is in general not in closed-form. We can use numerical integration to find it. For example, $h\left(\left(\tilde{\mathbf{F}}_{\mathbf{c}} \tilde{U}_{c}+\tilde{E}\right) \bmod A\right)$ can be computed as

$$
-\int_{\mathbf{g} \in\left[-\frac{A}{2} \frac{A}{2}\right]^{m}} f_{G}(\mathbf{g}) \log f_{G}(\mathbf{g}),
$$

where

$$
f_{G}(\mathbf{g})=2^{-n I_{c}} \sum_{\tilde{U}_{c}} \sum_{\mathbf{z} \in \mathbb{Z}^{m}} f_{\tilde{E}}\left(\mathbf{g}-\tilde{\mathbf{F}}_{\mathbf{c}} \tilde{U}_{c}-A \mathbf{z}\right),
$$

with $f_{\tilde{E}}(\cdot)$ being the PDF of the Gaussian random vector $\tilde{E}$ and $I_{c}$ being the bit per real symbol $\left(2^{I_{c}}\right.$ is the constellation size of $\tilde{U}_{c}$ ). If $A$ is large enough, the summation over all integer combinations $\mathbf{z} \in \mathbb{Z}^{m}$ can be reduced to over only few neighborhood terms. The other differential entropy term in (30) can be computed similarly. For the scheme using scalar quantizer described previously, similar expression can also be obtained using the distribution of $\tilde{E}$.

An alternative to test whether the selected argument $A$ in Section V-A is large enough is to see how close $h(\tilde{E} \bmod A)$ is to the Gaussian entropy $\frac{1}{2} \log (2 \pi e)^{m}\left|\Sigma_{\tilde{E}}\right|$. If $A$ is not large enough, a better TCQ has to be used to make it larger as described in Section V-A.

\section{End-to-End vector dirty paper coding structure}

The overall system block diagram is shown in Fig. 2. Without loss of generality, we focus on the equivalent real channel (3) and the achievement of (2). The transmitter consists of a nonsystematic IRA code, a TCQ with a mod- $A$ distortion measure, and linear transformations as in (8). The IRA encoder has an outer mixture of variable nodes (repetition codes, RC) with different degrees, an edge interleaver, an inner mixture of check nodes (single parity-check codes, SPC) with different degrees, and an accumulator (ACC) which is a memory one differential encoder. This code is nonsystematic since only the parity check bits are transmitted. If there are $N_{v}$ variable nodes and $N_{c}$ check nodes, the code rate of the IRA code $R_{c, R A}$ is $N_{v} / N_{c}$. After the constellation mapping with natural labeling, the constellation-mapped IRA coded bits are passed through the linear transform $\mathbf{F}_{\mathbf{c}}$. The rest of the transmitter in Fig. 2 follows (8). The mod- $A$ quantization error is transformed by $\mathbf{F}_{\mathbf{q}}$ to form the vector channel input signal $\mathbf{x}$. The vector interference channel is specified in (3).

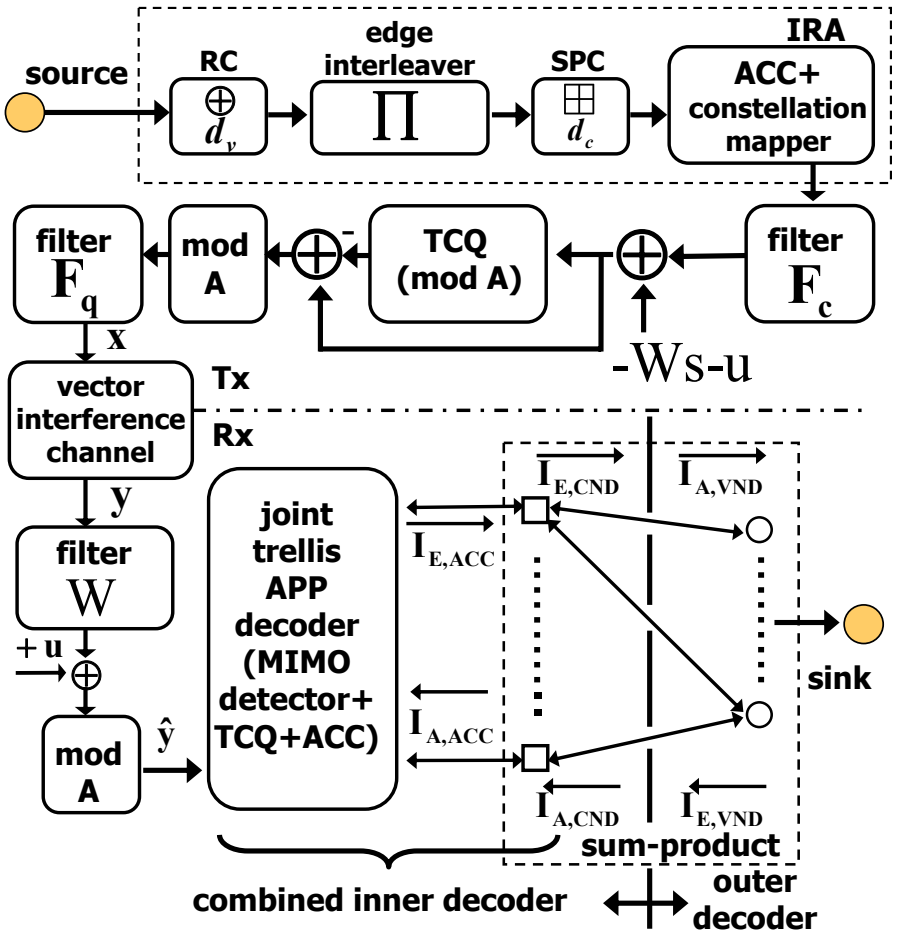

Fig. 2. Vector dirty paper coding with a nonsystematic irregular repeataccumulate codes (IRA), a trellis coded quantizer (TCQ) and iterative decoding.

At the receiver, after being equalized by $\mathbf{W}$ and removed the known dither, the signal is passed through an one-dimensional $\bmod -A$ operation to form $\hat{\mathbf{y}}$ in (11). Note that matrix filters $\mathbf{F}_{\mathbf{q}}, \mathbf{F}_{\mathbf{c}}$ and $\mathbf{W}$ are all block-diagonal and only operate in the spatial domain. Since the theoretical performance of the perfect successive decoding is hard to achieve in practice, we consider joint decoding of $\mathbf{c}_{\mathbf{c}}$ and $\mathbf{c}_{\mathbf{q}}$. Here we combine the MIMO detector, the TCQ decoder, and the ACC decoder into a joint trellis a posteriori probability (APP) decoder. The rest of the decoder contains a check node decoder (CND) and a variable node decoder (VND). The detail decoding algorithm of the joint trellis APP decoder and the sum-product algorithm used in the CND and VND can be found in Appendix II.

\section{A Design Example and Detailed Code PARAMETERS SELECTION}

In this section we provide a design example of the proposed vector DPC. The details of the parameter selection methods for the TCQ and IRA code will also be revealed. In order to achieve near theoretical performance, the number of symbols per codeword $T$ is typically very large ( $10^{5}$ order). Together with the multiple-transmitter antennas, the joint trellis APP decoder is very computationally intensive. To demonstrate our design with a reasonable simulation time, a 2 by 2 real MIMO channel is chosen as an example. However, the proposed architecture can be easily generalized to complex MIMO channels with more antennas.

We choose $R_{c}=n I_{c} R_{c, R A}=1$ bit per channel use. As an example, the channel matrix and the input covariance matrix are

$$
\mathbf{H}^{c}=\left[\begin{array}{ll}
1.7462 & 0.5161 \\
0.1180 & 2.2340
\end{array}\right], \quad \Sigma_{1}=\left[\begin{array}{ll}
0.1643 & 0.0790 \\
0.0790 & 0.3131
\end{array}\right] .
$$




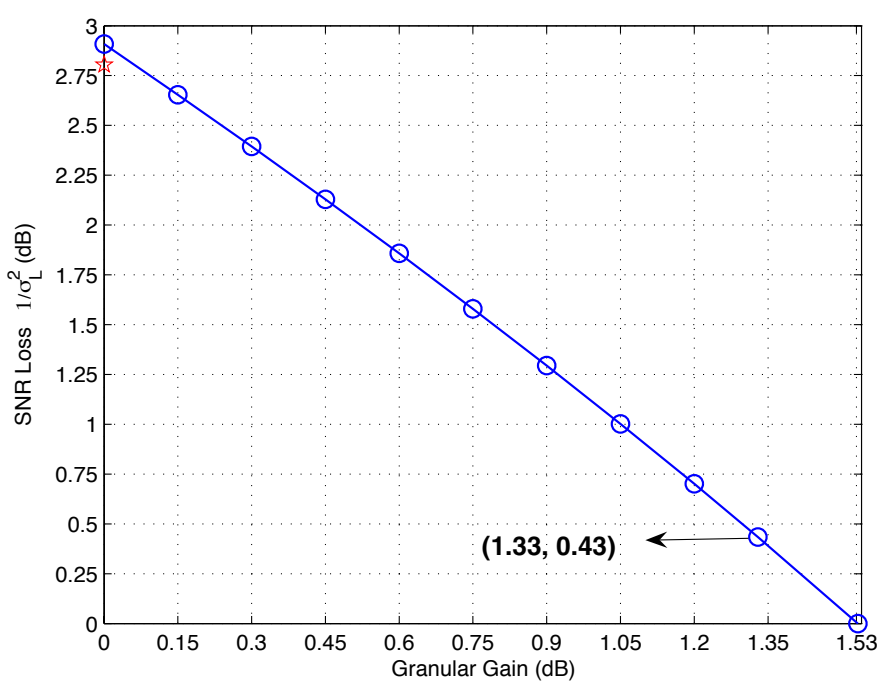

Fig. 3. Performance losses (28) due to practical TCQ under different granular gains. The value pointed by the arrow is the loss of the TCQ used in the simulation. The star indicates the loss of the scheme using a scalar quantizer (29).

It can be verified that $m=n=2$. This $\Sigma_{1}$ can achieve the Shannon limit $\left(C_{I F}=1 \mathrm{bit} / \mathrm{channel}\right.$ use $)$ of the real interference free channel $\mathbf{y}=\mathbf{H}^{c} \mathbf{x}+\mathbf{n}$ with power constraint $P_{1}=\operatorname{Tr}\left(\Sigma_{1}\right)=$ 0.4774 and unity noise variance. The real interference $\mathbf{s}$ is a bipolar phase shift keying (BPSK) signal sequence multiplied by $\mathbf{H}^{c}$. We obtain a $1.1 \mathrm{~dB}$ SNR loss compared to the Shannon limit through simulation. The performance loss is defined as the minimum amount the receiver noise variance $\sigma_{L}^{2}$ can be lowered while the decoder can decode with a small BER (around $10^{-6}$ ) at rate $R_{c}=C_{I F}$ with the transmission power $P_{1}$. That is, the $1.1 \mathrm{~dB}$ loss is due to lowering the noise variance from 1 to 0.77625 . The contributions of imperfect TCQ and IRA to this SNR loss will be discussed in the following subsections. This definition is also adopted in other DPC-related literatures [8], [14]-[16] and serves as a good performance comparison metric. In practice, lowering the receiver noise variance (or equivalently increasing the transmitted power) may cause the channel input covariance matrix for each user and the user order to change. Dealing with such problems is beyond the scope of this paper.

\section{A. Vector quantizer parameters selection and performance}

The impact (28) of the given TCQ normalized second-order moment $G\left(C_{q}\right)$ on the SNR loss is first investigated. From (19), and the aforementioned definition of SNR loss, the lowered receiver noise variance $\sigma_{L}^{2}$ must satisfy

$$
C_{I F}^{L}+R_{q}^{o}=C_{I F}+R_{q}^{T},
$$

where $C_{I F}^{L}$ is the interference-free rate (2) when the noise variance is $\sigma_{L}^{2}$. Fig. 3 shows the SNR loss as a function of the granular gain [27] using (31) and (28). The granular gain is computed as $-10 \log \left(12 G\left(C_{q}\right)\right)$ and is zero for scalar quantizers. The rate-distortion bound achieving quantizer has a $1.53 \mathrm{~dB}$ granular gain and zero SNR loss. In the low granular gain region, this prediction might be inaccurate due to the non-Gaussianity of the transmitted signal. The SNR loss of the scheme utilizing the scalar quantizer described in section
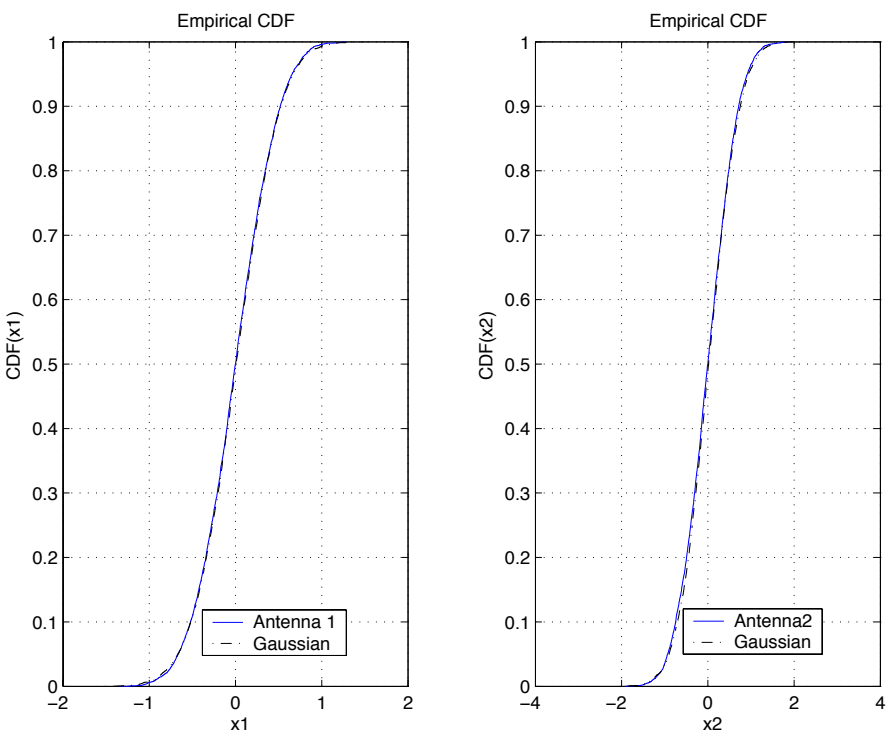

Fig. 4. The CDFs of the antenna outputs with the selected TCQ, each compared with the CDF of a Gaussian random variable with the same variance.

$\mathrm{V}$-A is also shown as a star in Fig. 3, and is slightly lower than the one predicted by (28) since the Gaussian vector has the maximum differential entropy between all random vectors with the same covariance matrix. The difference is only about $0.1 \mathrm{~dB}$.

We can determine the TCQ operating point according to the prediction in Fig 3. The selected TCQ parameters in the simulation are: code rate 1 bit per sample, number of states 256 , with feedforward polynomials $\left(515_{8}, 262_{8}\right)$. The LloydMax alphabet is used. It is possible to obtain better alphabets with exhaustive computer search [26]. The quantization-input to quantization-error SNR $1 / D_{q}$ is $7.35 \mathrm{~dB}$, using the mod- $A$ distortion measure. The value is measured with 100 distortion samples as in [26]. Using (24), $A$ is 8.0766 and the TCQ input range is [-4.0383 4.0383]. Note that $D_{q}$ is independent of the TCQ input range. The granular gain is $1.33 \mathrm{~dB}$ using (27). According to Fig. 3, this indicates that the noise variance must be lowered by about $0.4 \mathrm{~dB}$ compared to the ideal case.

We now investigate the transmitter output characteristics using the selected TCQ. By choosing $\mathbf{F}_{\mathbf{q}}$ satisfying (12), the simulated transmitter covariance matrix $\Sigma_{1}$, averaged over $2 * 10^{5}$ TCQ input samples, is

$$
\left[\begin{array}{ll}
0.1642 & 0.0789 \\
0.0789 & 0.3132
\end{array}\right]
$$

which is very close to the desired one specified before. The total transmission power is 0.4774 , which meets the power constraint $P_{1}$. Another important issue is to make the transmitter output distributed like Gaussian. As explained in Section IV, if the TCQ is good enough and $A$ is large enough, the transmitter output distribution will become Gaussian according to the rate-distortion test channel. Our TCQ has a granular gain that is only $0.2 \mathrm{~dB}$ lower than the optimal value. As shown in Fig. 4, the cumulative distribution functions (CDF) of the two transmitter antenna outputs are very close to Gaussian. 


\section{B. Channel coding parameters selection and performance}

We first find the proper constellation size by testing if (30 can reach $C_{I F}$ at the lowered noise variance $\sigma_{L}^{2}$ found ir the previous subsection. As a result, the BPSK constellatior suffices for our setting. The IRA code rate $R_{c, R A}$ is set tc be $1 / 2$. Also, a design margin of $0.7 \mathrm{~dB}$ is added in additior to the $0.4 \mathrm{~dB}$ quantizer induced loss, due the non-perfec IRA channel code. Note that this constellation size selectior method does not consider the decoder structure. A mor accurate method is using the area under the mutual informatior transfer curve (EXIT) of the joint trellis APP decoder [15] The EXIT curve for a soft input/soft output decoder is the transformation from its input a-priori information to its outpu extrinsic information. The area under the EXIT curve 0 the joint trellis APP decoder is an estimate of the mutua information of the ACC output bits and $\hat{\mathbf{y}}$. However, thi: method is more computation-time consuming compared with (30), which can be computed with conventional numerical integration packages or the ensemble average (Monte-Carlo method). Thus (30) can serve as a quick search of proper constellation size when the quantizer is good enough.

The rest of the IRA design is to find its edge distributions. We use the EXIT curve fitting technique in [15], [30]. As indicted in Fig. 2, we combine the joint-trellis APP decoder and the CND as the inner decoder, while treating the VND as the outer decoder. Curve fitting means to match the outer VND EXIT curve $I_{E, V N D}\left(I_{A, V N D}\right)$ to the inner TCQ\&ACC\&CND curve $I_{E \text {,inner }}\left(I_{A, \text { inner }}\right)$, that is,

$$
I_{E, \text { inner }}(I) \simeq I_{E, V N D}^{-1}(I), \forall 0 \leq I \leq 1,
$$

where $I_{E, V N D}^{-1}(\cdot)$ means the inverse function of the VND EXIT curve. To ensure the iterative decoding converge at low error rate, the outer VND EXIT curve must entirely lie under the inner TCQ\&ACC\&CND curve except at $(1,1)$,

$$
I_{E, \text { inner }}(I)>I_{E, V N D}^{-1}(I), \forall 0 \leq I<1 .
$$

The edge distributions of the IRA code will change the inner and outer EXIT curves. The EXIT curves of the individual variable node and check node decoder with certain degree can be obtained analytically [30]. The EXIT curve of the joint trellis APP decoder is found by computer simulation, where the a-priori L-values are modeled as Gaussian distributed. The details of how to obtain the EXIT curves of the code mixtures to find $I_{E \text {,inner }}($.$) and I_{E, V N D}($.$) can be found in [30].$

The EXIT curve fitting at the target noise variance are shown in Fig. 5. Here we use the biregular CND [30] (only two kinds of degree for the check nodes and one degree is 1). The CND profile is : $20 \%$ of nodes with degree 1 and $80 \%$ degree 3 ; the VND profile is : $23.89 \%$ degree $2,65.9 \%$ degree 4, $10.09 \%$ degree 18 and $0.12 \%$ degree 225 . From the L-value arithmetic (41) in Appendix II, a node of the CND outputs a non-zero L-value only when all input L-values are non-zero. Thus only the degree 1 check nodes (no coding is added) will pass the L-values from the joint-trellis APP decoder to the VND at the first iteration. This ensures that the inner EXIT curve starts from a non-zero point when no a-priori L-values are provided, and makes convergence possible. The IRA input block length $N_{v}$ is chosen to be $10^{5}$. The BER reaches $10^{-6}$

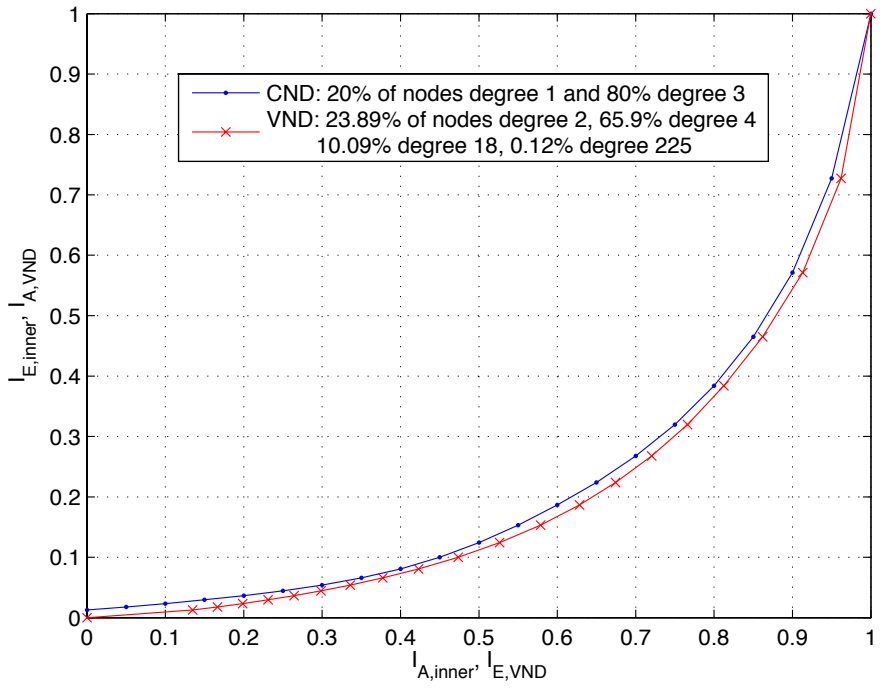

Fig. 5. EXIT chart of the rate $R_{c, R A}=1 / 2$ nonsystematic IRA code after curve fitting of the inner TCQ,ACC,CND\&BPSK curve and outer VND curve. Noise variance $\sigma_{L}^{2}$ is lowered by $1.1 \mathrm{~dB}$ from the ideal unity value. Input codeword length $N_{v}=10^{5}$ bits.

after $1.5 * 10^{6}$ input bits ( 15 blocks) are simulated. The decoder converges between 64-103 iterations.

Since our joint-trellis APP processer outputs extrinsic information high enough to trigger the convergence at the first iteration, systematic doping [15] is not used. The random interleaver is uniformly generated, and no parallel edges are allowed to avoid the length two circle. Since there are no apriori information of the variable nodes at the first iteration, a few inner iterations of sum-product decoding between the CND and VND will help to increase the $I_{E, V N D}$ at the first round.

\section{CONCLUSION}

In this paper, we have proposed an optimal vector dirty paper coding structure for the MIMO GBC where the jointtransmitter sends information to users without receiver cooperation. In particular, we extended the superposition dirty paper coding approach developed by Bennatan et al. [14] to the vector channel. Through a theoretical random coding analysis, the resultant coding structure was shown to be able to achieve the promised rate of this channel. The code was also implemented with existing practical vector quantizer (TCQ) and capacity-achieving IRA code. The design example validated our theory, which had similar performance compared with the best reported results for the scalar DPC channel [14][16].

One obvious future work is to reduce the receiver decoding complexity. Inherited from [14], the receiver indeed deals with a two-user MAC where the quantization and channel codes need to be decoded. Thus successive decoding or other decoding techniques developed for the MAC may bring complexity reduction for our scheme. Moreover, the channel code is the same as the code in [30] designed for the singleuser MIMO channel without channel feedback. Along with the vector quantizer structure, our receiver blocks are very similar to those in [30]. This similarity may be further explored to 
develop a reconfigurable coding architecture for both channels, which is favorable to hardware designers.

\section{APPENDIX I}

\section{PROOF OF THEOREM 1 AND ITS COROLLARY}

In this random-coding based proof, we start by defining the following random vectors, with their superscripts denoting the corresponding dimensions divided by $T$ to simply notations:

- $U_{A}^{m}$ is a random vector uniformly distributed over $[-A / 2, A / 2]^{m T}$, which is the Voronoi region [28] of the $m T$-dimensional cubic lattice $A \mathbf{Z}^{m T}$.

- $\tilde{X}_{G}^{m} \sim N_{\mathbb{R}}\left(0, \mathbf{I}_{m T}\right)$ and $U_{c}^{n} \sim N_{\mathbb{R}}\left(0, \mathbf{I}_{n T}\right)$ are real zero-mean white Gaussian random vectors with each element having unit variance.

- All other capital random vectors represent their corresponding signals (denoted in lower case previously) in the random-coding analysis. For example, $X^{2 M}$ corresponds to $\mathbf{x}$.

In the above, the first three random vectors and the interference $S^{r}$, which is not necessarily Gaussian, are independent. The random codebook for $C_{C}$ is formed by generating the $2^{T R_{c}}$ codewords according to the same distribution as $U_{c}^{n}$, with each codeword generated independently. Similarly, the random codebook for $C_{q}$ is obtained by generating the $2^{T R_{q}}$ codewords according to a distribution $U_{q}^{m}$ defined as

$$
U_{q}^{m}=\left[\left(-\mathbf{F}_{\mathbf{c}} U_{c}^{n}+\mathbf{W} S^{r}+U_{A}^{m}\right)+\tilde{X}_{G}^{m}\right] \bmod A .
$$

\section{A. Proof of the encoder part}

Following [14], we use the jointly typical encoder-decoder instead of the minimum-distance metric in this random coding analysis. The encoder is then defined as

Encoder: The encoder selects a codeword $\mathbf{c}_{\mathbf{c}} \in \mathcal{C}_{c}$ according to the message index, and finds a quantizer output $\mathbf{c}_{\mathbf{q}} \in \mathcal{C}_{q}$, such that the pair $\mathbf{c}_{\mathbf{q}}$ and $\left(-\mathbf{F}_{\mathbf{c}} \mathbf{c}_{\mathbf{c}}+\mathbf{W s}+\mathbf{u}\right) \bmod A$ are jointly strongly $\varepsilon$-typical with respect to the distributions of $U_{q}^{m}$ and $\left(-\mathbf{F}_{\mathbf{c}} U_{c}^{n}+\mathbf{W} S^{r}+U_{A}^{m}\right) \bmod A$. If no such codeword is found, an encoder error happens. Otherwise the transmitter transmits the vector $\mathbf{x}=\mathbf{F}_{\mathbf{q}} \tilde{\mathbf{x}}$, where

$$
\tilde{\mathbf{x}}=\left(\mathbf{c}_{\mathbf{q}}+\mathbf{F}_{\mathbf{c}} \mathbf{c}_{\mathbf{c}}-\mathbf{W s}-\mathbf{u}\right) \bmod A .
$$

Note that this encoder is similar to the one in the proof of the rate-distortion theory [11] [32, Sec. 4.4]. We can treat $\left(-\mathbf{F}_{\mathbf{c}} U_{c}^{n}+\mathbf{W} S^{r}+U_{A}^{m}\right) \bmod A$ as the random quantizer input and $\tilde{X}_{G}^{m} \bmod A$ as the quantization noise. Note that (32) also specifies the test channel since $U_{q}^{m}$ is independent of $\tilde{X}_{G}^{m} \bmod A$. This independence results from (32), the independence between $U_{c}^{n}, S^{r}, \tilde{X}_{G}^{m}$ and the dither $U_{A}^{m}$ by definition and the Lemma 1 of [28]. Therefore as in [14], the encoder error probability approaches zero with $T$ if

$$
R_{q}-\varepsilon_{1}>\frac{1}{T} I\left(U_{q}^{m} ;\left(-\mathbf{F}_{\mathbf{c}} U_{c}^{n}+\mathbf{W} S^{r}+U_{A}^{m}\right) \bmod A\right),
$$

where $\varepsilon_{1}$ is some small value and approaches 0 with $\varepsilon$. The RHS of (33) equals to

$$
\begin{aligned}
& \frac{1}{T}\left\{h\left(U_{q}^{m}\right)-h\left(U_{q}^{m} \mid\left(-\mathbf{F}_{\mathbf{c}} U_{c}^{n}+\mathbf{W} S^{r}+U_{A}^{m}\right) \bmod A\right)\right\} \\
& =2 \tilde{M} \log A-\frac{1}{T} h\left(\tilde{X}_{G}^{m} \bmod A\right) \\
& =2 \tilde{M} \log A-\tilde{M} \log (2 \pi e)+\delta_{1},
\end{aligned}
$$

where $h(\cdot)$ denotes the differential entropy of its argument and $\tilde{M}=m / 2$. For $h\left(U_{q}^{m}\right)$ in the first equality, note that the mod$A$ operation equals to the modulo- $\Lambda$ operation defined in [28] with $\Lambda=A \mathbf{Z}^{m T}$. By observing the definitions of the dither $U_{A}^{m}$ and $U_{q}^{m}$ in (32), we have that $U_{q}^{m}$ is uniformly distributed over $[-A / 2, A / 2]^{m T}$ with differential entropy $m T \log A$, according to Lemma 1 of [28]. The second term of the first equality comes from the selection of $U_{q}^{m}$ in (32), and the independence between $\tilde{X}_{G}^{m}$ and the quantizer input by definition. Since as $A$ increases, $\tilde{X}^{m}=\left(\tilde{X}_{G}^{m} \bmod A\right)$ approaches $\tilde{X}_{G}^{m}[31], \delta_{1}$ in the second equality approaches 0 as $A \rightarrow \infty$. With small enough $\varepsilon$ (then $\varepsilon_{1}$ ) and large enough $A,(33)$ is met and the encoder error probability will approach zero by the selection of $R_{q}$ in (18).

Now we check the satisfaction of the transmitter power constraint $\mathbb{E}\left\|X^{2 M}\right\|^{2} / T \leq P_{1}$. Since the linearly transformed quantization error is transmitted, $X^{2 M}=\mathbf{F}_{\mathbf{q}}\left(\tilde{X}_{G}^{m} \bmod A\right)$ and its covariance matrix is

$$
\Sigma_{x}=\mathbf{F}_{\mathbf{q}} \Sigma_{A}^{x} \mathbf{F}_{\mathbf{q}}^{\mathrm{T}},
$$

where $\Sigma_{A}^{x}$ is the covariance matrix of $\left(\tilde{X}_{G}^{m} \bmod A\right)$. Since the mod- $A$ operation is applied element-wise on the i.i.d random vector $\tilde{X}_{G}^{m}, \Sigma_{A}^{x}=P_{A} \mathbf{I}_{m}$, where $0<P_{A} \leq 1$ due to definition of the mod- $A$ operation. Using this fact and the preceding formula,

$$
\Sigma_{x}=P_{A} \mathbf{F}_{\mathbf{q}} \mathbf{F}_{\mathbf{q}}{ }^{\mathrm{T}}=P_{A} \Sigma_{G},
$$

and the power of $X^{2 M}$ is $\operatorname{Tr}\left(\Sigma_{x}\right) / T=P_{A} \cdot \operatorname{Tr}\left(\Sigma_{G}\right) / T=P_{A} \cdot P_{1}$. It is less than $P_{1}$, thus the power constraint is met. Furthermore, with the independence between $\tilde{X}_{G}^{m}$ and $S^{r}$ by selection, $X^{2 M}$ is independent of the multi-user interference $S^{r}$. This completes the proof of the encoder part of the theorem.

\section{B. Proof of the decoder part}

For the decoder, according to the channel (11), we focus on

$$
\hat{Y}^{m}=\left(U_{q}^{m}+\mathbf{F}_{\mathbf{c}} U_{c}^{n}+E^{m}\right) \bmod A,
$$

where $E^{m} \triangleq-\left(\mathbf{I}_{m T}-\mathbf{W} \tilde{\mathbf{H}}\right) \tilde{X}_{G}^{m}+\mathbf{W} N^{r}$, with $N^{r}$ defined as in (14). As in [14], we use the jointly-typical decoder

Decoder: The decoder finds $\hat{\mathbf{c}}_{\mathbf{c}} \in \mathcal{C}_{c}$ and $\hat{\mathbf{c}}_{\mathbf{q}} \in C_{q}$ such that $\left(\mathbf{F}_{\mathbf{c}} \hat{\mathbf{c}}_{\mathbf{c}}, \hat{\mathbf{c}}_{\mathbf{q}}, \hat{\mathbf{y}}\right)$ is jointly strongly $\varepsilon$-typical with respect to the distribution of $\left(\mathbf{F}_{\mathbf{c}} U_{c}^{n}, U_{q}^{m}, \hat{Y}^{m}\right)$.

Following [14], we know that the decoding error probability approaches zero with $T$ if

$$
R_{c}<\frac{1}{T} I\left(\mathbf{F}_{\mathbf{c}} U_{c}^{n} ; \hat{Y}^{m} \mid U_{q}^{m}\right), R_{q}<\frac{1}{T} I\left(U_{q}^{m} ; \hat{Y}^{m} \mid \mathbf{F}_{\mathbf{c}} U_{c}^{n}\right),
$$

$R_{c}+R_{q}<\frac{1}{T}\left(I\left(\mathbf{F}_{\mathbf{c}} U_{c}^{n}, U_{q}^{m} ; \hat{Y}^{m}\right)=I\left(U_{q}^{m} ; \hat{Y}^{m}\right)+I\left(\mathbf{F}_{\mathbf{c}} U_{c}^{n} ; \hat{Y}^{m} \mid U_{q}^{m}\right)\right)$. 
We first focus on $I\left(\mathbf{F}_{\mathbf{c}} U_{c}^{n} ; \hat{Y}^{m} \mid U_{q}^{m}\right) / T$, the maximum rate of $R_{c}$, and show that it equals to $C_{I F}+\delta_{2}$ in (19). From (35),

$$
\begin{aligned}
I\left(\mathbf{F}_{\mathbf{c}} U_{c}^{n} ; \hat{Y}^{m} \mid U_{q}^{m}\right) & =h\left(\hat{Y}^{m} \mid U_{q}^{m}\right)-h\left(\hat{Y}^{m} \mid U_{q}^{m}, \mathbf{F}_{\mathbf{c}} U_{c}^{n}\right) \\
& =h\left(\left(\mathbf{F}_{\mathbf{c}} U_{c}^{n}+E^{m}\right) \bmod A\right)-h\left(E^{m} \bmod A\right) .
\end{aligned}
$$

From (32), with the independence between $U_{c}^{n}, S^{r}, U_{A}^{m}$ and $\tilde{X}_{G}^{m}$ by definition, it is easy to show that $U_{q}^{m}$ is independent of $\mathbf{F}_{\mathbf{c}} U_{c}^{n}$ and $E^{m}$ by applying Lemma 1 of [28]. And the second equality comes from (35) and the independence between these random vectors. Moreover, the distribution of $\mathbf{F}_{\mathbf{c}} U_{c}^{n}+E^{m}$ is indeed the same as $\tilde{X}_{G}^{m}$. First note that by the definition of $E^{m}$, the LMMSE equalizer $\mathbf{W}$ makes $-E^{m}$ the LMMSE estimation error for estimating $\tilde{X}_{G}^{m}$ in channel (14). By the orthogonality principle [22], the covariance matrix of $E^{m}$ is $\left(\mathbf{I}_{m T}-\mathbf{W} \tilde{\mathbf{H}}\right)$. Furthermore, $E^{m}$ is also Gaussian and independent of $\mathbf{F}_{\mathbf{c}} U_{c}^{n}$. Together with (16), the distribution of the sum $\mathbf{F}_{\mathbf{c}} U_{c}^{n}+E^{m}$ is thus Gaussian with covariance matrix $\mathbf{W} \tilde{\mathbf{H}}+\left(\mathbf{I}_{m T}-\mathbf{W} \tilde{\mathbf{H}}\right)=$ $\mathbf{I}_{m T}$ which is the same as the distribution of $\tilde{X}_{G}^{m}$.

Substituting $h\left(\left(\mathbf{F}_{\mathbf{c}} U_{c}^{n}+E^{m}\right) \bmod A\right)$ with $h\left(\tilde{X}_{G}^{m} \bmod A\right)$ in (37),

$$
\begin{aligned}
\frac{1}{T} I\left(\mathbf{F}_{\mathbf{c}} U_{c}^{n} ; \hat{Y}^{m} \mid U_{q}^{m}\right) & =\frac{1}{T}\left(h\left(\tilde{X}_{G}^{m} \bmod A\right)-h\left(E^{m} \bmod A\right)\right) \\
& =\frac{1}{T}\left(h\left(\tilde{X}_{G}^{m}\right)-h\left(E^{m}\right)\right)+\delta_{2},
\end{aligned}
$$

where $\delta_{2} \rightarrow 0$ as $A \rightarrow \infty$ since the aliasing effects of the modulo operation are negligible. Since

$$
h\left(\tilde{X}_{G}^{m}\right)-h\left(E^{m}\right)=\frac{1}{2} \log \frac{\left|\mathbf{I}_{m T}\right|}{\left|\Sigma_{E}\right|},
$$

where $\Sigma_{E}$ is the covariance matrix of the LMMSE error $-E^{m}$, and this conditional mutual information is the maximum achievable rate of the backward-channel $\tilde{X}_{G}^{m}=\mathbf{W} Y_{f}^{r}-E^{m}$ corresponding to the forward-channel (14), by invoking the information-lossless property [22], the maximum achievable rate is

$$
\frac{1}{2 T} \log \frac{\left|\mathbf{I}_{m T}\right|}{\left|\Sigma_{E}\right|}=\frac{1}{T} I\left(\tilde{X}_{G}^{m} ; Y_{f}^{r}\right)=\frac{1}{T}\left\{h\left(Y_{f}^{r}\right)-h\left(N^{r}\right)\right\} .
$$

Using the fact that the covariance matrix of $N^{r}$ in (14) is $\frac{1}{2} \mathbf{I}_{r T}$ and (12), we arrive at

$$
\begin{aligned}
\frac{1}{T}\left(h\left(\tilde{X}_{G}^{m}\right)-h\left(E^{m}\right)\right) & =\frac{1}{2 T} \log \left(\left|\tilde{\mathbf{H}} \tilde{\mathbf{H}}^{\mathrm{T}}+\frac{1}{2} \mathbf{I}_{r T}\right| /\left|\frac{1}{2} \mathbf{I}_{r T}\right|\right) \\
& =\frac{1}{2 T} \log \left(\left|\mathbf{H}\left(2 \Sigma_{G}\right) \mathbf{H}^{\mathrm{T}}+\mathbf{I}_{r T}\right| /\left|\mathbf{I}_{r T}\right|\right) .
\end{aligned}
$$

It is easy to show that this rate equals to the $C_{I F}$ in (2) with complex notations. The proof of the $R_{c}$ upper bound in (19) is completed.

For the sum rate capacity in (36), since we have shown that $I\left(\mathbf{F}_{\mathbf{c}} U_{c}^{n} ; \hat{Y}^{m} \mid U_{q}^{m}\right) / T=C_{I F}+\delta_{2}$, we focus on $I\left(U_{q}^{m} ; \hat{Y}^{m}\right) / T$. Interestingly, $I\left(U_{q}^{m} ; \hat{Y}^{m}\right) / T$ equals to $R_{q}^{o}+\delta_{1}$ in (18) and the sum rate upper bound in (19) follows. From Lemma 1 of [28] and (35), $\hat{Y}^{m}$ is uniformly distributed over $[-A / 2, A / 2]^{m T}$ by treating $U_{q}^{m}$ as the dither. Therefore,

$$
\begin{aligned}
\frac{1}{T} I\left(U_{q}^{m} ; \hat{Y}^{m}\right) & =\frac{1}{T}\left\{h\left(\hat{Y}^{m}\right)-h\left(\left(\mathbf{F}_{\mathbf{c}} U_{c}^{n}+E^{m}\right) \bmod A \mid U_{q}^{m}\right)\right\} \\
& =2 \tilde{M} \log A-\frac{1}{T} h\left(\left(\mathbf{F}_{\mathbf{c}} U_{c}^{n}+E^{m}\right) \bmod A\right) .
\end{aligned}
$$

Note that $h\left(\left(\mathbf{F}_{\mathbf{c}} U_{c}^{n}+E^{m}\right) \bmod A\right)=h\left(\tilde{X}_{G}^{m} \bmod A\right)$ as in the previous derivation. Compared with (34), we conclude that $R_{q}^{o}+\delta_{1}$ is equal to $\frac{1}{T} I\left(U_{q}^{m} ; \hat{Y}^{m}\right)$. The sum rate capacity is the sum of (39) and (37), which equals to

$$
\frac{1}{T} I\left(U_{q}^{m}, \mathbf{F}_{\mathbf{c}} U_{c}^{n} ; \hat{Y}^{m}\right)=2 \tilde{M} \log A-\frac{1}{T} h\left(E^{m} \bmod A\right) .
$$

Surprisingly, this rate equals to the upper-bound of $R_{q}$ in (36), thus the $R_{q}$ rate constraint can be removed. To see this,

$$
\begin{aligned}
\frac{1}{T} I\left(U_{q}^{m} ; \hat{Y}^{m} \mid \mathbf{F}_{\mathbf{c}} U_{c}^{n}\right) & =\frac{1}{T}\left\{h\left(\hat{Y}^{m} \mid \mathbf{F}_{\mathbf{c}} U_{c}^{n}\right)-h\left(\hat{Y}^{m} \mid U_{q}^{m}, \mathbf{F}_{\mathbf{c}} U_{c}^{n}\right)\right\} \\
& =\frac{1}{T}\left(h\left(U_{q}^{m}+E^{m} \bmod A\right)-h\left(E^{m} \bmod A\right)\right) \\
& \left.=2 \tilde{M} \log A-\frac{1}{T} h\left(E^{m} \bmod A\right)\right),
\end{aligned}
$$

where $U_{q}^{m}$ is treated as dither in $\left(U_{q}^{m}+E^{m}\right) \bmod A$ again.

Finally, we know that as $A \rightarrow \infty, \quad\left(R_{q}^{o}, C_{I F}\right)=$ $\left(I\left(U_{q}^{m} ; \hat{Y}^{m}\right) / T, I\left(\mathbf{F}_{\mathbf{c}} U_{c}^{n} ; \hat{Y}^{m} \mid U_{q}^{m}\right) / T\right)$ is at the vertex of the MAC region specified in the theorem, thus successive decoding is possible. And this concludes our proof.

\section{Proof of Corollary 1}

The dithers will make all transmitted signals independent of each other. If $A_{k} \rightarrow \infty, 1 \leq k \leq K$, then following the previous derivations we know that all transmitted signals will approach Gaussian and make $E_{j}^{m}$ Gaussian. The interference-free rate is achievable. Moreover, $\Sigma_{n_{j}}$ becomes

$$
\frac{1}{2} \mathbf{I}_{2 N_{j} T}+\sum_{k<j} \mathbf{H}_{j} \Sigma_{G, k} \mathbf{H}_{j}^{\mathrm{T}},
$$

and the interference-free rate is equal to $R_{c, j}$ specified in the corollary.

\section{APPENDIX II \\ Receiver Decoding Algorithms}

For the joint trellis APP decoder, the BCJR algorithm [33], [34] is applied and the details are presented in the next subsection. This block computes the extrinsic information of the ACC input bits and passes them to the CND comprising the $N_{c}$ check nodes. The CND then computes the L-values and passes them through the edge de-interleaver to the outer VND comprising $N_{v}$ variable nodes. The VND uses these Lvalues to find new $\mathrm{L}$-values that are then fed back to the CND. Then the CND will generate new L-values accordingly, and feeds back them to the joint-trellis APP decoder as the apriori information of the ACC input bits. These complete one round of the iterative decoding. The VND and CND L-value operations follow the well-known sum-product algorithm [30]. The L-value arithmetic for a node of the VND is $L_{i, \text { out }}=$ $\sum_{j \neq i} L_{j, i n}$, where $L_{j, i n}$ is the $j$ th a-priori L-value of the edge going into this node and $L_{i, \text { out }}$ is the $i$ th extrinsic L-value of the edge coming out of this node. For the CND node, the input/output L-value arithmetic is

$$
L_{i, \text { out }}=\ln \frac{1-\prod_{j \neq i} \tanh \left(-\frac{L_{j, \text { in }}}{2}\right)}{1+\prod_{j \neq i} \tanh \left(-\frac{L_{j, \text { in }}}{2}\right)} .
$$




\section{A. Joint trellis processing}

As in [15], we combine the accumulator trellis (memory one) and TCQ trellis (memory $m_{T C Q}$ ) to form a joint trellis. Moreover, we can also combine this joint trellis decoding with the modulo symbol metric based MIMO detector as in [30]. This trellis has $2^{1+m_{T C Q}}$ states and $2^{n I_{c}+m I_{q}}$ edges per state, if the TCQ has $I_{q}$ bits per sample. Each edge is labelled by the $n I_{c}$ bits which indicate the vector IRA channel coded symbol, together with the $m I_{q}$ bits which indicate the TCQ output. The state transition is determined by running $n I_{c}$ bits through the ACC memory and $m I_{q}$ bits through the TCQ memory. Here we assume that $I_{q}$ is an integer for simplicity. It is easy to generalize to the non-integer case [26].

The APP decoder computes the a-posteriori L-value on each accumulator input bit as

$$
L\left(b_{A C C, k} \mid \hat{\mathbf{y}}\right)=\ln \frac{P\left(b_{A C C, k}=+1 \mid \hat{\mathbf{y}}\right)}{P\left(b_{A C C, k}=-1 \mid \hat{\mathbf{y}}\right)},
$$

where $b_{A C C, k}$ is the $k$ th bit of the ACC input sequence, $0 \leq k<$ $\left(n I_{c}\right) T-1$. Due to the effect of the dither, all TCQ-codewords are treated equally likely and independent of the information bits as in [15]. We can rewrite the above equation as

$$
L\left(b_{A C C, k} \mid \hat{\mathbf{y}}\right)=\ln \frac{\sum_{\mathbf{c}_{\mathbf{q}} \in C_{q}} P\left(b_{A C C, k}=+1 \mid \hat{\mathbf{y}}, \mathbf{c}_{\mathbf{q}}\right)}{\sum \mathbf{c}_{\mathbf{q}} \in C_{q} P\left(b_{A C C, k}=-1 \mid \hat{\mathbf{y}}, \mathbf{c}_{\mathbf{q}}\right)} .
$$

The right hand side can be divided into the well-known apriori and extrinsic parts and computed efficiently by the BCJR algorithm. The log-MAP BCJR algorithm is adopted for its numerical precision and stability. After removing some constant terms, we can simplify the log-likelihood function $\ln f\left(\hat{\mathbf{y}}_{t} \mid \mathbf{c}_{\mathbf{q}, \mathbf{t}}, \mathbf{c}_{\mathbf{c}, \mathbf{t}}\right)$ for a certain edge of the $t$ th processed received symbol $\hat{\mathbf{y}}_{t}$ as

$$
\ln \sum_{\mathbf{z} \in \mathbb{Z}^{m}} \exp \left(-\frac{1}{2}\left(\mathbf{e}^{\prime}\right)^{\mathbf{T}} \Sigma_{\tilde{E}}^{-1} \mathbf{e}^{\prime}\right)
$$

where

$$
\mathbf{e}^{\prime}=\left(\hat{\mathbf{y}}_{t}-\mathbf{c}_{\mathbf{q}, \mathbf{t}}-\tilde{\mathbf{F}}_{\mathbf{c}} \mathbf{c}_{\mathbf{c}, \mathbf{t}}-A \mathbf{z}\right),
$$

with $\Sigma_{\tilde{E}}$ and $\tilde{\mathbf{F}}_{\mathbf{c}}$ defined in Section V-B. The hypothesized IRA channel coded vector symbol $\mathbf{c}_{\mathbf{c}, \mathbf{t}}$ depends on the starting state of this edge and the information bits running through the ACC memory indicated by the edge label, while the hypothesized TCQ output symbol $\mathbf{c}_{\mathbf{q}, \mathbf{t}}$ depends on the bits running through the TCQ memory, respectively. If $A$ is large enough, only a few neighbor terms are needed in the summation. Moreover, we can use the "Jacobian logarithm" [34] to further reduce the computational complexity. The a-priori information of ACC input sequences is provided by the CND decoder. Note that no a-priori information is provided for the TCQ sequences.

\section{ACKNOWLEDGEMENTS}

The authors would like to thank Prof. Daniel P. Palomar and the reviewers for their suggestions that greatly improve the presentation of the paper. They would also like to thank PinHsun Lin and Wen-Hsien Chu for their helpful discussions, and Jing-Ye Yu and Wan-Yi Lin for the help on programming.

\section{REFERENCES}

[1] H. Weingarten, Y. Steinberg, and S. Shamai, "The capacity region of the Gaussian multiple-input multiple-output broadcast channel," IEEE Trans. Inform. Theory, vol. 52, no. 9, pp. 3936 - 3964, Sept. 2006.

[2] S. Vishwanath, N. Jindal, and A. Goldsmith, "Duality, achievable rates, and sum-rate capacity of Gaussian MIMO broadcast channels," IEEE Trans. Inform. Theory, vol. 49, no. 10, pp. 2658 - 2668, Oct. 2003.

[3] W. Yu and J. M. Cioffi, "Sum capacity of Gaussian vector broadcast channels," IEEE Trans. Inform. Theory, vol. 50, no. 9, pp. 1875-1892, Sept. 2004.

[4] P. Viswanath and D. Tse, "Sum capacity of the vector Gaussian broadcast channel and uplink-downlink duality," IEEE Trans. Inform. Theory, vol. 49, no. 8, pp. 1912-1921, 2003.

[5] N. Jindal, U. Mitra, and A. Goldsmith, "Capacity of Ad-Hoc networks with node cooperation," in Proceedings of International Symposium on Information Theory, (ISIT), Chicago, USA, Jun. 2004, p. 271.

[6] M. Costa, "Writing on dirty paper," IEEE Trans. Inform. Theory, vol. 29, no. 3, pp. 439 - 441, May 1983.

[7] C. Peel, B. Hochwald, and A. Swindlehurst, "A vector-perturbation technique for near-capacity multiantenna multiuser communication-part I: channel inversion and regularization," IEEE Trans. Commun., vol. 53, no. 3, pp. 195- 202, Jan. 2005.

[8] B. M. Hochwald, C. B. Peel, and A. L. Swindlehurst, "A vector-perturbation technique for near-capacity multiantenna multiuser communication-part II: perturbation," IEEE Trans. Commun., vol. 53, no. 3, pp. 537-544, Jan. 2005.

[9] C. Windpassinger, R. Fischer, T. Vencel, and J. Huber, "Precoding in multiantenna and multiuser communications," IEEE Trans. Wireless Commun., vol. 3, no. 4, pp. 1305-1316, Jul. 2004.

[10] A. Amraoui, G. Kramer, and S. Shamai, "Coding for the MIMO broadcast channel," in in Proc. IEEE International Symposium on Information Theory, Yokohama, Japan, June 2003, p. 296.

[11] T. Cover and J. Thomas, Elements of Information Theory. New York: Wiley, 1991.

[12] M. Tomlinson, "New automatic equalizer employing modulo arithmetic," Electron. Lett., vol. 7, pp. 138-139, Mar. 1971.

[13] R. Zamir, S. Shamai, and U. Erez, "Nested linear/lattice codes for structured multiterminal binning," IEEE Trans. Inform. Theory, vol. 48, no. 6, pp. 1250-1276, June 2002.

[14] A. Bennatan, D. Burshtein, G. Caire, and S. Shamai, "Superposition coding for side-information channels," IEEE Trans. Inform. Theory, vol. 52, no. 5, pp. 1872 - 1889, May 2006.

[15] U. Erez and S. ten Brink, "A close to capacity dirty paper coding scheme," IEEE Trans. Inform. Theory, vol. 51, no. 10, pp. 3417-3432, Oct. 2005.

[16] Y. Sun, A. Liveris, V. Stankovic, and Z. Xiong, "Near-capacity dirtypaper code design: A source-channel coding approach," in Proc. 39th Annual Conference on Information Sciences and System (CISS 2005), Baltimore, MD, March 2005, Mar. 2005.

[17] N. Jindal, W. Rhee, S. Vishwanath, S. A. Jafar, and A. Goldsmith, "Sum power iterative water-filling for multi-antenna Gaussian broadcast channels," IEEE Trans. Inform. Theory, vol. 51, no. 4, pp. 1570 - 1580, Apr. 2005.

[18] W. Yu, A. Sutivang, D. Julian, T. Cover, and M. Chiang, "Writing on colored paper," in Proc. of IEEE International Symposium on Information Theory (ISIT), Jun. 2001, p. 302.

[19] R. A. Horn and C. R. Johnson, Matrix analysis. Cambridge, UK: Cambridge university press, 1985.

[20] R. M. Gray and D. L. Neuhoff, "Quantization," IEEE Trans. Inform. Theory, vol. 44, no. 6, pp. 2325-2383, Oct. 1998.

[21] S. Y. Chung, "On the construction of some capacity-approaching coding schemes," Ph.D. dissertation, Massachusetts Institute of Technology, Sep. 2000.

[22] G. D. Forney, Jr., "Shannon meets Wiener II: On MMSE estimation in successive decoding schemes," in 42th Annual Allerton Conf. on Comm. Control, and Comput., Monticello, IL, Sep. 2004.

[23] U. Erez, S. Shamai, and R. Zamir, "Capacity and lattice strategies for canceling known interference," IEEE Trans. Inform. Theory, vol. 51, no. 11 , pp. 3820 - 3833, Nov 2005.

[24] H. E. Gamal, G. Caire, and M. O. Damen, "Lattice coding and decoding achieve the optimal diversity-vs-multiplexing tradeoff of MIMO channels," IEEE Trans. Inform. Theory, vol. 50, no. 6, pp. 968 - 985, Jun 2004.

[25] S. C. Lin and H. J. Su, "Lattice-based vector dirty paper coding for MIMO Gaussian broadcast channels," in the 5th Asia-Europe Workshop on Information Theory (AEW), Oct.29-Nov.1 2006. 
[26] M. W. Marcellin and T. R. Fischer, "Trellis coded quantization of memoryless and Gauss-Markov sources," IEEE Trans. Commun., vol. 38, no. 1, pp. 82-93, Jan. 1990.

[27] M. Eyuboglu and G. D. Forney, Jr., "Lattice and trellis quantization with lattice- and trellis-bounded codebooks-high-rate theory for memoryless sources," IEEE Trans. Inform. Theory, vol. 39, no. 1, pp. 46-59, Jan. 1993.

[28] U. Erez and R. Zamir, "Achieving 1/2 log (1+SNR) on the AWGN channel with lattice encoding and decoding," IEEE Trans. Inform. Theory, vol. 50, no. 10, pp. 2293-2314, Oct. 2004.

[29] H. Jin, A. Khandekar, and R. McEliece, "Irregular repeat-accumulate codes," in Proc. 2nd Int. Symp. Turbo Codes Related Topics, Brest,France, Sept. 2000, pp. $1-8$.

[30] S. ten Brink and G. Kramer, "Turbo processing for scalar and vector channels," in Proc. 3rd Int. Symp. Turbo Codes Related Topics, Brest, France, Sept. 2003, pp. 23- 30.

[31] G. D. Forney, Jr., M. D. Trott, and S. Y. Chung;, "Sphere-boundachieving coset codes and multilevel coset codes," IEEE Trans. Inform. Theory, vol. 46, no. 3, pp. 820 - 850, May 2000.

[32] T. Berger, Rate distortion theory. Englewood Cliffs, NJ: Prentice-Hall, 1971.

[33] L. Bahl, J. Cocke, F. Jelinek, and J. Raviv, "Optimal decoding of linear codes for minimizing symbol error rate," IEEE Trans. Inform. Theory, vol. 20, pp. 284-287, Mar. 1974.

[34] P. Robertson, E. Villebrun, and P. Hoeher, "A comparison of optimal and suboptimal map decoding algorithms operating in the log domain," in Proc. Int. Conf. Communications, Jun. 1995, pp. 1009-1013.

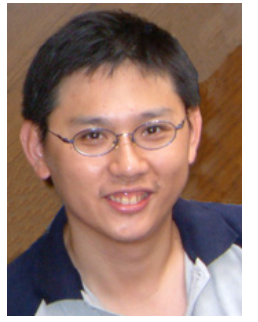

Shih-Chun Lin received his B.S. degree from the Department of Electrical Engineering at the National Taiwan University, Taipei, Taiwan, in 2000. Since 2004, he has been working towards his Ph.D. degree in the Graduate Institute of Communication Engineering at the National Taiwan University. He was with the Graduate Institute of Electronics Engineering at the same university between 2000-2004. His research interests include information theory, signal processing and communications.

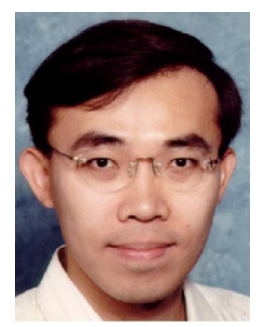

Hsuan-Jung Su received the B.S. degree in Electronics Engineering from the National Chiao-Tung University, Taiwan, in 1992, and the M.S. and $\mathrm{Ph} . \mathrm{D}$. degrees in Electrical Engineering from the University of Maryland, College Park, in 1996 and 1999, respectively. From 1999 to 2000, he was a Postdoctoral Research Associate with the Institute for Systems Research, University of Maryland. From 2000 to 2003, he was with the Bell Laboratories, Lucent Technologies, Holmdel, New Jersey, where he was involved in the design and performance evaluation of adaptive coding/modulation, fast Hybrid-ARQ, scheduling, and Radio Link Control protocol for 3G wireless networks. In 2003, Dr. Su joined the Department of Electrical Engineering, National Taiwan University, where he is currently an Associate Professor. His research interests cover coding, modulation, signal processing, power control and synchronization of narrowband and wideband communication systems. 